\title{
QUANTITATIVE PHOTOACOUSTIC IMAGING IN THE RADIATIVE TRANSPORT REGIME*
}

\author{
ALEXANDER V. MAMONOV ${ }^{\dagger}$ AND KUI REN $\ddagger$
}

\begin{abstract}
The objective of quantitative photoacoustic tomography (QPAT) is to reconstruct optical and thermodynamic properties of heterogeneous media from data describing the absorbed energy distribution inside the media. There have been extensive theoretical and computational studies on the inverse problem in QPAT, although most were in the diffusive regime. We present in this work some numerical reconstruction algorithms for multi-source QPAT in the radiative transport regime with energy data collected at either single or multiple wavelengths. We show that when the medium to be probed is non-scattering, explicit reconstruction schemes can be derived to reconstruct the absorption and the Grüneisen coefficients. When data at multiple wavelengths are utilized, we can reconstruct simultaneously the absorption, scattering, and Grüneisen coefficients. We show by numerical simulations that the reconstructions are stable.
\end{abstract}

Key words. Quantitative photoacoustic tomography (QPAT), sectional photoacoustic tomography, radiative transport equation, inverse transport problem, interior data, Born approximation, iterative reconstruction.

AMS subject classifications. 49N45, 65M 32, 74J25, 92C55.

\section{Introduction}

In photoacoustic tomography (PAT) experiments, we send near infra-red (NIR) light into a biological tissue. The tissue absorbs part of the incoming light and heats up due to the absorbed energy. The heating then results in expansions of the tissue and the expansion generates compressive (acoustic) waves. We then measure the time-dependent acoustic signal that arrives on the surface of the tissue. From the knowledge of these acoustic measurements, we are interested in reconstructing the absorption and scattering properties of the tissue, as well as the thermodynamic Grüneisen parameter which measures the photoacoustic efficiency of the tissue. We refer interested readers to $[4,12,22,28,33,59,61,67,72,95,96,100]$ for overviews of the field of photoacoustic imaging.

The propagation of NIR light in biological tissues is accurately modeled by the radiative transport equation, which describes the distribution of photons in the phase space. To be precise, let $\Omega \in \mathbb{R}^{d}(d \geq 2)$ be the domain of interest with smooth boundary $\partial \Omega$ and $\mathbb{S}^{d-1}$ be the unit sphere in $\mathbb{R}^{d}$. We denote by $X=\Omega \times \mathbb{S}^{d-1}$ the phase space and $\Gamma_{ \pm}=\left\{(\mathbf{x}, \mathbf{v}):(\mathbf{x}, \mathbf{v}) \in \partial \Omega \times \mathbb{S}^{d-1}\right.$ s.t. $\left.\pm \mathbf{n}(\mathbf{x}) \cdot \mathbf{v}>0\right\}$ its incoming and outgoing boundaries, $\mathbf{n}(\mathbf{x})$ being the unit outer normal vector at $\mathbf{x} \in \partial \Omega$. The radiative transport equation for photon density $u(\mathbf{x}, \mathbf{v})$ can then be written as $[9,11,77]$ :

$$
\begin{aligned}
\mathbf{v} \cdot \nabla u(\mathbf{x}, \mathbf{v})+\sigma_{a}(\mathbf{x}) u(\mathbf{x}, \mathbf{v}) & =\sigma_{s}(\mathbf{x}) K(u)(\mathbf{x}, \mathbf{v}), & & \text { in } X, \\
u(\mathbf{x}, \mathbf{v}) & =g(\mathbf{x}, \mathbf{v}), & & \text { on } \Gamma_{-},
\end{aligned}
$$

Here the positive functions $\sigma_{a}(\mathbf{x})$ and $\sigma_{s}(\mathbf{x})$ are the absorption and the scattering coefficients, respectively. The function $g(\mathbf{x}, \mathbf{v})$ is the incoming illumination source.

*Received: July 19, 2012; accepted (in revised form): January 5, 2013. Communicated by Lenya Ryzhik.

${ }^{\dagger}$ Institute for Computational Engineering and Sciences, University of Texas, Austin, TX 78712, USA (mamonov@ices.utexas.edu).

${ }^{\ddagger}$ Department of Mathematics, University of Texas, Austin, TX 78712, USA (ren@math.utexas.edu). 
The scattering operator $K$ is defined as

$$
K(u)(\mathbf{x}, \mathbf{v})=\int_{\mathbb{S}^{d-1}} \mathcal{K}\left(\mathbf{v}, \mathbf{v}^{\prime}\right) u\left(\mathbf{x}, \mathbf{v}^{\prime}\right) d \mathbf{v}^{\prime}-u(\mathbf{x}, \mathbf{v}),
$$

where $d \mathbf{v}$ is the normalized measure on $\mathbb{S}^{d-1}$ in the sense that $\int_{\mathbb{S}^{d-1}} d \mathbf{v}=1$, and the kernel $\mathcal{K}\left(\mathbf{v}, \mathbf{v}^{\prime}\right)$ describes the way that photons traveling in direction $\mathbf{v}^{\prime}$ are scattered into direction $\mathbf{v}$, and satisfies the normalization condition $\int_{\mathbb{S}^{d-1}} \mathcal{K}\left(\mathbf{v}, \mathbf{v}^{\prime}\right) d \mathbf{v}^{\prime}=1, \forall \mathbf{v} \in$ $\mathbb{S}^{d-1}$. In practical applications in biomedical optics, $\mathcal{K}$ is often taken to be the HenyeyGreenstein phase function $[9,54,97]$, which depends only on the product $\mathbf{v} \cdot \mathbf{v}^{\prime}$; see equation (5.5) in Section 5.

The photon energy that is absorbed at location $\mathbf{x} \in \Omega$ per unit volume, $E(\mathbf{x})$, is the product of the absorption coefficient and the fluence distribution:

$$
E(\mathbf{x})=\int_{\mathbb{S}^{d-1}} \sigma_{a}(\mathbf{x}) u(\mathbf{x}, \mathbf{v}) d \mathbf{v} .
$$

The heating due to this absorbed energy generates an initial pressure field, denoted by $H$, in the tissue that depends on the thermodynamic properties of the tissue and is proportional to $E$ :

$$
H(\mathbf{x})=\Upsilon(\mathbf{x}) E(\mathbf{x}) \equiv \Upsilon(\mathbf{x}) \int_{\mathbb{S}^{d-1}} \sigma_{a}(\mathbf{x}) u(\mathbf{x}, \mathbf{v}) d \mathbf{v},
$$

where the positive function $\Upsilon(\mathbf{x})$ is the nondimensional Grüneisen coefficient which in the current formulation measures the photoacoustic efficiency of the tissue. To simplify the presentation, in the rest of this work we use the short notation $\langle f\rangle_{\mathbf{v}}$ to denote the integral of $f$ over the $\mathbf{v}$ variable.

The initial pressure field $H$ then propagates according to the acoustic wave equation, with the wave speed $c(\mathbf{x})$,

$$
\begin{array}{cc}
\frac{1}{c^{2}(\mathbf{x})} \frac{\partial^{2} p}{\partial t^{2}}-\Delta p=0, & \text { in } \mathbb{R}_{+} \times \mathbb{R}^{d}, \\
p(0, \mathbf{x})=H(\mathbf{x}), \quad \frac{\partial p}{\partial t}(0, \mathbf{x})=0, & \text { in } \mathbb{R}^{d} .
\end{array}
$$

The time-dependent pressure signal $p(t, \mathbf{x})$ is then measured on the surface of the tissue for long enough time, say $t_{\infty}$, and the objective is to reconstruct the coefficients $\sigma_{a}, \sigma_{s}$, and the Grüneisen coefficient $\Upsilon$ from this measurement. Note that the reason that we can write the transport equation in the stationary case while using the wave equation in the time-dependent case is that the two phenomena occur on two significantly different time scales [13].

The reconstruction problem in photoacoustic tomography can be split into two steps. In the first step, we need to reconstruct the initial pressure field $H(\mathbf{x})$ from the measured acoustic signal on the boundary, $\left.p(t, \mathbf{x})\right|_{(0, T) \times \partial \Omega}$. This is a well known inverse problem for the acoustic wave equation that has been thoroughly studied in the past a few years under various scenarios; see for instance $[1,2,24,29,40,42,41$, $49,50,51,52,53,60,64,65,62,63,68,69,72,82,99]$ for analytical reconstruction formulas with constant wave speed, $[55,56,73,85,88,89,98]$ for reconstruction under variable wave speed, and $[7,8,36,58,91,92,3,23,32,36,71,70,73,87,86,90,101]$ for reconstructions under even more complicated situations.

This work is concerned with the second step of photoacoustic tomography, called quantitative photoacoustic tomography (QPAT). The objective is to reconstruct the 
absorption and the diffusion coefficients, $\sigma_{a}$ and $\sigma_{s}$, in the transport equation (1.1) and the Grüneisen coefficient $\Upsilon$ from the result of the first step, the data $H$ in (1.3). This step has recently attracted significant attention from mathematical $[5,6,13,21$, 14, 15, 16, 18, 19], computational [20, 30, 31, 34, 48, 44, 66, 80, 81, 83, 104, 106], and modeling and experimental $[17,75]$ perspectives. Most of existing work on this step, however, is done in the diffusive regime $[14,16,18]$, i.e., it is based on the diffusion approximation to the transport equation (1.1). Transport-based QPAT is only studied in $[13,34,102]$ where the Grüneisen coefficient $\Upsilon$ has been assumed to be a known constant.

To set up the problem appropriately, for the rest of the paper, we assume that all the coefficients that we are interested in are positive and bounded functions. More precisely, we assume:

(A1) the coefficients $0<c_{0} \leq \Upsilon(\mathbf{x}), \sigma_{a}(\mathbf{x}), \sigma_{s}(\mathbf{x}) \in L^{1}(\Omega) \cap L^{\infty}(\Omega)$ for some $c_{0}>0$;

(A2) the scattering kernel $\mathcal{K}\left(\mathbf{v}, \mathbf{v}^{\prime}\right) \in L^{1}\left(\mathbb{S}^{d-1} \times \mathbb{S}^{d-1}\right)$;

(A3) the illumination, modeled by the boundary condition $g$, is in $L^{1}\left(\Gamma_{-}, d \boldsymbol{\xi}\right)$, with measure $d \boldsymbol{\xi}=|\mathbf{v} \cdot \mathbf{n}(\mathbf{x})| d \mathfrak{m}(\mathbf{x}) d \mathbf{v}, d \mathfrak{m}(\mathbf{x})$ being the usual Lebesgue measure on $\partial \Omega$.

With these assumptions, it is well-known that the radiative transport problem (1.1) is well-posed and thus admits a unique solution $u \in L^{1}(X)$ [35]. This means that the data $H$ in (1.3) is a well-defined function in $L^{1}(\Omega)$. In practical applications, the objects to be imaged are often embedded into phantoms of similar optical and acoustic properties to get regularly shaped imaging domains. We thus assume that the domain $\Omega$ is convex. This assumption will simplify some of the presentation but is not essential for the results obtained.

We conclude this section with two remarks. First, if both the absorption coefficient $\sigma_{a}(\mathbf{x})$ and the scattering coefficient $\sigma_{s}(\mathbf{x})$ are known and only the Grüneisen coefficient must be reconstructed, we can simply solve the transport equation (1.1) and compute the energy $E(\mathbf{x})$. Then $\Upsilon(\mathbf{x})$ is reconstructed as $\Upsilon=\frac{H}{E}$. Thus we need only one interior data set and one transport solver to solve the inverse problem. This is a trivial case. We will not discuss this case in the rest of the paper.

Second, in practical application of PAT in biomedical imaging, the absorption and the scattering coefficients $\sigma_{a}$ and $\sigma_{s}$ are often isotropic, i.e. independent of the angular variable $\mathbf{v}$. We thus restrict ourselves to the case of isotropic coefficients in this work. Mathematically this assumption is essential, as we can see from the following result that it is not possible to uniquely reconstruct anisotropic coefficients.

Proposition 1.1. Let $\left(\Upsilon, \sigma_{a}, \sigma_{s}\right)$ and $\left(\tilde{\Upsilon}, \tilde{\sigma}_{a}, \sigma_{s}\right)$ be two sets of coefficients, and $H$ and $\tilde{H}$ the corresponding data sets. Let $z(\mathbf{x}) \in \mathcal{C}^{1}(\bar{\Omega})$ be an arbitrary positive function with boundary value $\left.z\right|_{\partial \Omega}=1$. Then

$$
\tilde{\sigma}_{a}=\left(\sigma_{a}-\mathbf{v} \cdot \nabla \ln z\right) z \quad \text { and } \quad \tilde{\Upsilon} \tilde{\sigma}_{a}=\Upsilon \sigma_{a}
$$

implies $\tilde{H}=H$.

Proof. Let $u$ be the solution of the transport equation for coefficients $\left(\sigma_{a}, \sigma_{s}\right)$ with boundary value $g$. It is straightforward to verify that $u z$ is the solution of the transport equation for coefficients $\left(\sigma_{a}-\mathbf{v} \cdot \nabla \ln z, \sigma_{s}\right)$ with the same boundary value $g$ (because $\left.z\right|_{\partial \Omega}=1$ ). Now, clearly $\tilde{\Upsilon} \int_{\mathbb{S}^{d-1}}\left(\sigma_{a}-\mathbf{v} \cdot \nabla \ln z\right) u z d \mathbf{v}=\Upsilon \int_{\mathbb{S}^{d-1}} \sigma_{a} u d \mathbf{v}$ if (1.5) holds. 
The rest of the paper is structured as follows. We first study in Section 2 the inverse transport problem for non-scattering media for applications in quantitative sectional QPAT. We present some analytical reconstruction strategies in this setting. We then study in Section 3 the same inverse problem for scattering media. We linearize the nonlinear inverse problem using the tool of Born approximation and present some numerical procedures to solve the linear and nonlinear inverse problems. In Section 4 we consider the QPAT problem in the case when illuminations with multiple wavelengths are available. To validate the reconstruction strategies, in Section 5 we provide several numerical simulations with synthetic data generated under different scenarios. We conclude the paper with some additional remarks in Section 6 .

\section{QPAT of non-scattering media}

We start by considering the QPAT problem for non-scattering media. In this case, photons propagate in the media along straight trajectories, without changing their propagation directions. The scattering mechanism in the transport equation (1.1) is thus dropped by setting the scattering coefficient $\sigma_{s}=0$. We have the following transport model for light propagation:

$$
\begin{aligned}
\mathbf{v} \cdot \nabla u(\mathbf{x}, \mathbf{v})+\sigma_{a}(\mathbf{x}) u(\mathbf{x}, \mathbf{v}) & =0, & & \text { in } X, \\
u(\mathbf{x}, \mathbf{v}) & =g(\mathbf{x}, \mathbf{v}), & & \text { on } \Gamma_{-} .
\end{aligned}
$$

The fact that photons travel in straight lines allows us to illuminate only part of the domain, for instance a plane cut through a three-dimensional medium. This is the fundamental principle of sectional photoacoustic tomography [107, 39].

To simplify the presentation, for any point $\mathbf{x} \in \Omega$ and direction $\mathbf{v} \in \mathbb{S}^{d-1}$, let us define

$$
\tau_{ \pm}(\mathbf{x}, \mathbf{v})=\inf \left\{s \in \mathbb{R}_{+} \mid \mathbf{x} \pm s \mathbf{v} \notin \Omega\right\}
$$

and $\tau(\mathbf{x}, \mathbf{v})=\tau_{+}(\mathbf{x}, \mathbf{v})+\tau_{-}(\mathbf{x}, \mathbf{v})$. It is easy to see that $\tau_{+}(\mathbf{x}, \mathbf{v})\left(\operatorname{resp} . \tau_{-}(\mathbf{x}, \mathbf{v})\right)$ is the distance it takes a particle at $\mathbf{x}$ traveling in direction $\mathbf{v}($ resp. $-\mathbf{v})$ to reach the boundary of the domain. In the same spirit, for any point $(\mathbf{x}, \mathbf{v})$ on the incoming boundary $\Gamma_{-}$, we define

$$
\tau_{+}(\mathbf{x}, \mathbf{v})=\sup \left\{s \in \mathbb{R}_{+} \mid \mathbf{x} \pm s \mathbf{v} \in \Omega\right\},
$$

and set $\tau_{-}(\mathbf{x}, \mathbf{v})=0$. Thus $\tau_{+}(\mathbf{x}, \mathbf{v})$ is the distance for a photon coming into the domain at $\mathbf{x}$ in direction $\mathbf{v}$ to exit the domain.

It is straightforward to show the following representation of the interior data.

Lemma 2.1. The interior data $H(\mathbf{x})$ generated with source $g$ and coefficients $\left(\Upsilon, \sigma_{a}\right)$ can be written as

$$
H(\mathbf{x})=\Upsilon(\mathbf{x}) \sigma_{a}(\mathbf{x}) \int_{\mathbb{S}^{d-1}} g\left(\mathbf{x}-\tau_{-}(\mathbf{x}, \mathbf{v}) \mathbf{v}, \mathbf{v}\right) e^{-\int_{0}^{\tau_{-}(\mathbf{x}, \mathbf{v})} \sigma_{a}\left(\mathbf{x}-\tau_{-}(\mathbf{x}, \mathbf{v}) \mathbf{v}+s \mathbf{v}\right) d s} d \mathbf{v} .
$$

Proof. The transport equation (2.1) can be integrated along direction $\mathbf{v}$ as an ODE to obtain the solution:

$$
u(\mathbf{x}, \mathbf{v})=g\left(\mathbf{x}-\tau_{-}(\mathbf{x}, \mathbf{v}) \mathbf{v}, \mathbf{v}\right) e^{-\int_{0}^{\tau_{-}(\mathbf{x}, \mathbf{v})} \sigma_{a}\left(\mathbf{x}-\tau_{-}(\mathbf{x}, \mathbf{v}) \mathbf{v}+s \mathbf{v}\right) d s} .
$$

The result then follows from using this solution in the data $H$ introduced in (1.3).

In fact, this simple representation of the transport solution allows us to obtain an explicit procedure for reconstructing the two unknown coefficients $\Upsilon$ and $\sigma_{a}$ if we have the luxury of using the right illumination source $g(\mathbf{x}, \mathbf{v})$. 

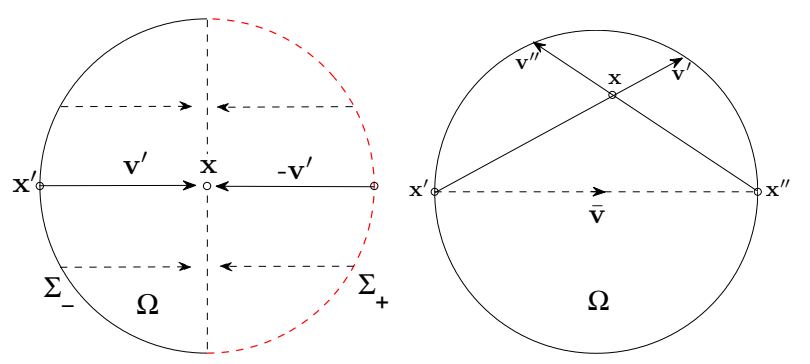

FIG. 2.1. Two illumination schemes that allow the simultaneous reconstruction of $\Upsilon$ and $\sigma_{a}$ using two data sets in a circular domain. Left: two collimated sources supported on $\Sigma_{-}$(solid part of $\partial \Omega$ ) and $\Sigma_{+}$(dashed part of $\left.\partial \Omega\right)$, respectively; Right: two point sources located at $\mathbf{x}^{\prime}$ and $\mathbf{x}^{\prime \prime}$, respectively.

2.1. Reconstruction with collimated sources. The first case where we can derive an analytical reconstruction formula is when collimated sources, i.e., sources focused on a specific direction, are used. Let $\mathbf{v}^{\prime}$ be the direction in which the source points, and define $\Sigma_{ \pm}\left(\mathbf{v}^{\prime}\right) \equiv\left\{\mathbf{x} \in \partial \Omega \mid \pm \mathbf{n}(\mathbf{x}) \cdot \mathbf{v}^{\prime}>0\right\}, \Sigma_{-}\left(\mathbf{v}^{\prime}\right)$ (resp. $\left.\Sigma_{+}\left(\mathbf{v}^{\prime}\right)\right)$ to be the part of the boundary $\partial \Omega$ where lines in direction $\mathbf{v}^{\prime}$ enters (resp. leaves) the domain $\Omega$. As mentioned in the introduction, we assume that the domain $\Omega$ is convex. In this case, each point $\mathbf{x} \in \Sigma_{-}\left(\mathbf{v}^{\prime}\right)$ is uniquely mapped to a point $\mathbf{y} \in \Sigma_{+}\left(\mathbf{v}^{\prime}\right)$ as: $\mathbf{y}=\mathbf{x}+\tau_{+}\left(\mathbf{x}, \mathbf{v}^{\prime}\right) \mathbf{v}^{\prime}$, and vise versa: $\mathbf{x}=\mathbf{y}+\tau_{+}\left(\mathbf{y},-\mathbf{v}^{\prime}\right)\left(-\mathbf{v}^{\prime}\right)$.

The collimated illumination source that we choose has the form

$$
g(\mathbf{x}, \mathbf{v})=\mathfrak{g}(\mathbf{x}) \delta\left(\mathbf{v}-\mathbf{v}^{\prime}\right), \quad \mathbf{x} \in \Sigma_{-}\left(\mathbf{v}^{\prime}\right) .
$$

This leads to the following expression for the interior data:

$$
H(\mathbf{x})=\mathfrak{g}\left(\mathbf{x}-\tau_{-}\left(\mathbf{x}, \mathbf{v}^{\prime}\right) \mathbf{v}^{\prime}\right)\left(\Upsilon(\mathbf{x}) \sigma_{a}(\mathbf{x}) e^{-\int_{0}^{\tau_{-}\left(\mathbf{x}, \mathbf{v}^{\prime}\right)} \sigma_{a}\left(\mathbf{x}-\tau_{-}\left(\mathbf{x}, \mathbf{v}^{\prime}\right) \mathbf{v}^{\prime}+s \mathbf{v}^{\prime}\right) d s}\right) .
$$

If $\mathbf{x}^{\prime} \in \partial \Omega$ is the back-track of $\mathbf{x}$ to the boundary in $-\mathbf{v}$ direction, i.e., $\mathbf{x}^{\prime}=\mathbf{x}-$ $\tau_{-}\left(\mathbf{x}, \mathbf{v}^{\prime}\right) \mathbf{v}^{\prime}$, then $H$ can be written as

$$
\frac{H\left(\mathbf{x}^{\prime}+\tau_{-}\left(\mathbf{x}, \mathbf{v}^{\prime}\right) \mathbf{v}^{\prime}\right)}{\mathfrak{g}\left(\mathbf{x}^{\prime}\right) \Upsilon\left(\mathbf{x}^{\prime}+\tau_{-}\left(\mathbf{x}, \mathbf{v}^{\prime}\right) \mathbf{v}^{\prime}\right)}=\sigma_{a}\left(\mathbf{x}^{\prime}+\tau_{-}\left(\mathbf{x}, \mathbf{v}^{\prime}\right) \mathbf{v}^{\prime}\right) e^{-\int_{0}^{\tau_{-}\left(\mathbf{x}, \mathbf{v}^{\prime}\right)} \sigma_{a}\left(\mathbf{x}^{\prime}+s \mathbf{v}^{\prime}\right) d s}
$$

(i) Reconstruction of $\sigma_{a}$. If $\Upsilon$ is known, then we can take the log of the above formula and differentiate with respect to $\tau_{-}$to obtain the following equation for $\sigma_{a}$ :

$$
\begin{aligned}
\mathbf{v}^{\prime} \cdot \nabla \sigma_{a}(\mathbf{x})-\sigma_{a}^{2}(\mathbf{x})-\left(\mathbf{v}^{\prime} \cdot \nabla \ln \frac{H}{\Upsilon \mathfrak{g}}\right) \sigma_{a}(\mathbf{x}) & =0, \quad \text { in } \Omega, \\
\sigma_{a}(\mathbf{x}) & =\frac{H(\mathbf{x})}{\Upsilon(\mathbf{x}) \mathfrak{g}(\mathbf{x})}, \quad \text { on } \Sigma_{-},
\end{aligned}
$$

where $\mathfrak{g}$ in the term $\mathbf{v}^{\prime} \cdot \nabla \ln \frac{H}{\Upsilon_{\mathfrak{g}}}$ is evaluated at $\mathbf{x}-\tau_{-}\left(\mathbf{x}, \mathbf{v}^{\prime}\right) \mathbf{v}^{\prime}$. This is an initial value problem for a first order ordinary differential equation (because the direction $\mathbf{v}^{\prime}$ is fixed). It can be solved uniquely [43] to reconstruct the absorption coefficient $\sigma_{a}$ along the lines in direction $\mathbf{v}^{\prime}$.

There is an equivalent reconstruction procedure for $\sigma_{a}$. When a collimated source is used, photons travel only along the direction to which the source is pointing, say 
$\mathbf{v}^{\prime}$. Thus, the solution $u$ to the transport equation is only non-zero in this direction. In other words, $\langle u\rangle_{\mathbf{v}} \equiv \int_{\mathbb{S}^{d-1}} u(\mathbf{x}, \mathbf{v}) d \mathbf{v}=u\left(\mathbf{x}, \mathbf{v}^{\prime}\right)$, and hence the data can be expressed as $H=\Upsilon \sigma_{a}(\mathbf{x}) u\left(\mathbf{x}, \mathbf{v}^{\prime}\right)$. We can thus replace the absorption term $\sigma_{a}(\mathbf{x}) u(\mathbf{x}, \mathbf{v})$ in the transport equation with the term $H / \Upsilon$ to obtain the following transport equation for the photon density $u$ :

$$
\begin{aligned}
\mathbf{v} \cdot \nabla u(\mathbf{x}, \mathbf{v})+\frac{H}{\Upsilon} & =0, & & \text { in } X, \\
u(\mathbf{x}, \mathbf{v}) & =\mathfrak{g}(\mathbf{x}) \delta\left(\mathbf{v}-\mathbf{v}^{\prime}\right), & & \text { on } \Gamma_{-} .
\end{aligned}
$$

This transport equation can be solved uniquely for $u$ [35]:

$$
u\left(\mathbf{x}^{\prime}+t \mathbf{v}, \mathbf{v}\right)= \begin{cases}\mathfrak{g}\left(\mathbf{x}^{\prime}\right)-\int_{0}^{t} \frac{H}{\Upsilon}\left(\mathbf{x}^{\prime}+s \mathbf{v}\right) d s, & \mathbf{x}^{\prime} \in \Sigma_{-} \text {and } \mathbf{v}=\mathbf{v}^{\prime} \\ 0, & \mathbf{x}^{\prime} \in \Sigma_{-} \text {and } \mathbf{v} \neq \mathbf{v}^{\prime}\end{cases}
$$

Once $u$ is obtained, we can reconstruct the absorption $\sigma_{a}=H /(\Upsilon u)$ provided that we select the boundary condition $\mathfrak{g}$ such that the denominator does not vanish.

(ii) Reconstruction of $\left(\Upsilon, \sigma_{a}\right)$. In fact, we can use two data sets generated with collimated sources to determine the Grüneisen coefficient and the absorption coefficients simultaneously. The configuration of the two sources is illustrated in figure 2.1 (left). Let $g_{1}(\mathbf{x}, \mathbf{v})=\mathfrak{g}(\mathbf{x}) \delta\left(\mathbf{v}-\mathbf{v}^{\prime}\right)$ be the first collimated source supported on $\Sigma_{-}$, and $H_{1}$ be the corresponding interior data which takes the form of $H$ in (2.5). Then the second source we use is supported on $\Sigma_{+}$with an identical intensity distribution, except that it points in the opposite direction, i.e., $-\mathbf{v}^{\prime}$. More precisely,

$$
g_{2}(\mathbf{x}, \mathbf{v})=\mathfrak{g}\left(\mathbf{x}-\tau_{+}\left(\mathbf{x}, \mathbf{v}^{\prime}\right) \mathbf{v}^{\prime}\right) \delta\left(\mathbf{v}+\mathbf{v}^{\prime}\right), \quad \mathbf{x} \in \Sigma_{+}\left(\mathbf{v}^{\prime}\right)
$$

If we use this source in the expression for the data $H$ in (2.2), we obtain the following expression for the interior data $\mathrm{H}_{2}$ :

$$
\frac{H_{2}\left(\mathbf{x}^{\prime}+\tau_{-}\left(\mathbf{x}, \mathbf{v}^{\prime}\right) \mathbf{v}^{\prime}\right)}{\mathfrak{g}\left(\mathbf{x}^{\prime}\right) \Upsilon\left(\mathbf{x}^{\prime}+\tau_{-}\left(\mathbf{x}, \mathbf{v}^{\prime}\right) \mathbf{v}^{\prime}\right)}=\sigma_{a}\left(\mathbf{x}^{\prime}+\tau_{-}\left(\mathbf{x}, \mathbf{v}^{\prime}\right) \mathbf{v}^{\prime}\right) e^{-\int_{0}^{\tau_{+}\left(\mathbf{x}, \mathbf{v}^{\prime}\right)}} \sigma_{a}\left(\mathbf{x}^{\prime}+\tau\left(\mathbf{x}, \mathbf{v}^{\prime}\right) v^{\prime}-s \mathbf{v}^{\prime}\right) d s .
$$

We now take the logarithm of the ratio of (2.5) and (2.10) and use the relation $\tau(\mathbf{x}, \mathbf{v})=\tau_{+}(\mathbf{x}, \mathbf{v})+\tau_{-}(\mathbf{x}, \mathbf{v})$ to obtain

$$
\ln \frac{H_{2}}{H_{1}}\left(\mathbf{x}^{\prime}+\tau_{-}\left(\mathbf{x}, \mathbf{v}^{\prime}\right) \mathbf{v}^{\prime}\right)=-\int_{0}^{\tau_{+}\left(\mathbf{x}^{\prime}, \mathbf{v}^{\prime}\right)} \sigma_{a}\left(\mathbf{x}^{\prime}+s \mathbf{v}^{\prime}\right) d s+2 \int_{0}^{\tau_{-}\left(\mathbf{x}, \mathbf{v}^{\prime}\right)} \sigma_{a}\left(\mathbf{x}^{\prime}+s \mathbf{v}^{\prime}\right) d s .
$$

Differentiation of this result with respect to $\tau_{-}\left(\mathbf{x}, \mathbf{v}^{\prime}\right)$ (equivalent to the directional differentiation $\left.\mathbf{v}^{\prime} \cdot \nabla_{\mathbf{x}}\right)$ will allow us to reconstruct the quantity $2 \sigma_{a}\left(\mathbf{x}^{\prime}+\tau_{-}\left(\mathbf{x}, \mathbf{v}^{\prime}\right) \mathbf{v}^{\prime}\right)=$ $2 \sigma_{a}(\mathbf{x})$ a.e. The coefficient $\Upsilon$ can then be reconstructed by solving the transport equation with the reconstructed $\sigma_{a}$ and $g_{1}$ (resp. $\left.g_{2}\right)$, and computing $\Upsilon=H_{1} /\left(\sigma_{a}\left\langle u_{1}\right\rangle_{\mathbf{v}}\right)$ (resp. $\Upsilon=H_{2} /\left(\sigma_{a}\left\langle u_{2}\right\rangle_{\mathbf{v}}\right)$ ).

2.2. Reconstruction with point sources. The second case where we can derive an analytical reconstruction method is when the illumination source is a point source in the spatial variable:

$$
g(\mathbf{x}, \mathbf{v})=\mathfrak{g}(\mathbf{v}) \delta\left(\mathbf{x}-\mathbf{x}^{\prime}\right) .
$$


This is the most commonly used type of source in optical imaging, such as optical tomography [9]. For any point $\mathbf{x} \in \Omega$, let us define $\mathbf{v}^{\prime}=\frac{\mathbf{x}-\mathbf{x}^{\prime}}{\left|\mathbf{x}-\mathbf{x}^{\prime}\right|}$ as the unit vector pointing from $\mathbf{x}^{\prime}$ to $\mathbf{x}$. Then the interior data $H$ simplifies to

$$
H(\mathbf{x})=\mathfrak{g}\left(\mathbf{v}^{\prime}\right)\left(\Upsilon(\mathbf{x}) \sigma_{a}(\mathbf{x}) e^{-\int_{0}^{\tau_{-}\left(\mathbf{x}, \mathbf{v}^{\prime}\right)} \sigma_{a}\left(\mathbf{x}-\tau_{-}\left(\mathbf{x}, \mathbf{v}^{\prime}\right) \mathbf{v}^{\prime}+s \mathbf{v}^{\prime}\right) d s}\right) .
$$

Following the presentation in the previous section, we can rewrite this as

$$
\frac{H\left(\mathbf{x}^{\prime}+\tau_{-}\left(\mathbf{x}, \mathbf{v}^{\prime}\right) \mathbf{v}^{\prime}\right)}{\mathfrak{g}\left(\mathbf{v}^{\prime}\right) \Upsilon\left(\mathbf{x}^{\prime}+\tau_{-}\left(\mathbf{x}, \mathbf{v}^{\prime}\right) \mathbf{v}^{\prime}\right)}=\sigma_{a}\left(\mathbf{x}^{\prime}+\tau_{-}\left(\mathbf{x}, \mathbf{v}^{\prime}\right) \mathbf{v}^{\prime}\right) e^{-\int_{0}^{\tau_{-}\left(\mathbf{x}, \mathbf{v}^{\prime}\right)} \sigma_{a}\left(\mathbf{x}^{\prime}+s \mathbf{v}^{\prime}\right) d s} .
$$

This is the same type of formula as (2.5) except that the unit direction vectors $\mathbf{v}^{\prime}$ are different at different points $\mathbf{x} \in \Omega$.

(i) Reconstruction of $\sigma_{a}$. If $\Upsilon$ is known, we can differentiate (2.13) as before to obtain an equation for the absorption coefficient $\sigma_{a}$. For each $\mathbf{v}^{\prime} \in \mathbb{S}_{-}^{d-1}\left(\mathbf{x}^{\prime}\right) \equiv\{\mathbf{v} \in$ $\left.\mathbb{S}^{d-1} \mid \mathbf{n}\left(\mathbf{x}^{\prime}\right) \cdot \mathbf{v}<0\right\}$, define $\Omega_{\mathbf{v}^{\prime}} \equiv\left\{\mathbf{x} \in \Omega \mid \frac{\mathbf{x}-\mathbf{x}^{\prime}}{\left|\mathbf{x}-\mathbf{x}^{\prime}\right|}=\mathbf{v}^{\prime}\right\}$. Then

$$
\begin{aligned}
\mathbf{v}^{\prime} \cdot \nabla \sigma_{a}(\mathbf{x})-\sigma_{a}^{2}(\mathbf{x})-\left(\mathbf{v}^{\prime} \cdot \nabla \ln \frac{H(\mathbf{x})}{\Upsilon(\mathbf{x}) \mathfrak{g}\left(\mathbf{v}^{\prime}\right)}\right) \sigma_{a}(\mathbf{x}) & =0, & & \text { in } \Omega_{\mathbf{v}^{\prime}}, \\
\sigma_{a}(\mathbf{x}) & =\sigma_{a}\left(\mathbf{x}^{\prime}\right), & & \text { at } \mathbf{x}^{\prime} .
\end{aligned}
$$

For a fixed $\mathbf{v}^{\prime}$, we can solve this first order ODE to find $\sigma_{a}(\mathbf{x})$ along the line segment $\Omega_{\mathbf{v}^{\prime}}$ if we know the value of $\sigma_{a}(\mathbf{x})$ at $\mathbf{x}^{\prime}$. Due to the singularity of the source at $\mathbf{x}^{\prime}$, we can not reconstruct this value from the data $H$ as before.

Point sources emit photons that travel in straight lines away from the source location. Thus the transport solution at each spatial position $\mathbf{x}$ is only non-zero in direction $\mathbf{v}^{\prime}$. Hence $H=\Upsilon(\mathbf{x}) \sigma_{a}(\mathbf{x}) u\left(\mathbf{x}, \mathbf{v}^{\prime}\right)$. The transport equation can again be simplified to

$$
\begin{aligned}
\mathbf{v} \cdot \nabla u(\mathbf{x}, \mathbf{v})+\frac{H}{\Upsilon} & =0, & & \text { in } X \\
u(\mathbf{x}, \mathbf{v}) & =\mathfrak{g}(\mathbf{v}) \delta\left(\mathbf{x}-\mathbf{x}^{\prime}\right), & & \text { on } \Gamma_{-} .
\end{aligned}
$$

This transport equation can be conveniently solved in the polar coordinates with the origin at $\mathbf{x}^{\prime}$. The absorption coefficient can then be reconstructed as $\sigma_{a}=H /(\Upsilon u)$ away from $\mathbf{x}^{\prime}$.

(ii) Reconstruction of $\left(\Upsilon, \sigma_{a}\right)$. If both coefficients are unknown, we can use two data sets generated with point sources to reconstruct them. The setup is depicted in figure 2.1 (right). Let $g_{1}(\mathbf{x}, \mathbf{v})=\mathfrak{g}(\mathbf{v}) \delta\left(\mathbf{x}-\mathbf{x}^{\prime}\right)$ and $g_{2}(\mathbf{x}, \mathbf{v})=\mathfrak{g}(\mathbf{v}) \delta\left(\mathbf{x}-\mathbf{x}^{\prime \prime}\right)$ be the two point sources used to produce the interior data. We denote by $d\left(\mathbf{x}^{\prime}, \mathbf{x}^{\prime \prime}\right)=\left|\mathbf{x}^{\prime}-\mathbf{x}^{\prime \prime}\right|$ the distance between the two points and by $\overline{\mathbf{v}}=\frac{\mathbf{x}^{\prime \prime}-\mathbf{x}^{\prime}}{\left|\mathbf{x}^{\prime \prime}-\mathbf{x}^{\prime}\right|}$ the unit vector pointing from $\mathbf{x}^{\prime}$ to $\mathbf{x}^{\prime \prime}$. For any point $\mathbf{x} \in \Omega$, we define $\mathbf{v}^{\prime \prime}=\frac{\mathbf{x}-\mathbf{x}^{\prime \prime}}{\left|\mathbf{x}-\mathbf{x}^{\prime \prime}\right|}$ as the unit vector pointing from $\mathrm{x}^{\prime \prime}$ to $\mathbf{x}$. It is then straightforward to verify that

$$
\tau_{-}\left(\mathbf{x}, \mathbf{v}^{\prime}\right) \mathbf{v}^{\prime} \cdot \overline{\mathbf{v}}+\tau_{-}\left(\mathbf{x}, \mathbf{v}^{\prime \prime}\right) \mathbf{v}^{\prime \prime} \cdot(-\overline{\mathbf{v}})=d\left(\mathbf{x}^{\prime}, \mathbf{x}^{\prime \prime}\right) .
$$

The first source $g_{1}$ produces data $H_{1}$ that is given in (2.13), while the second source $g_{2}$ produces the data $H_{2}$ that is given by

$$
H_{2}(\mathbf{x})=\mathfrak{g}\left(\mathbf{v}^{\prime \prime}\right)\left(\Upsilon(\mathbf{x}) \sigma_{a}(\mathbf{x}) e^{-\int_{0}^{\tau_{-}\left(\mathbf{x}, \mathbf{v}^{\prime \prime}\right)} \sigma_{a}\left(\mathbf{x}-\tau_{-}\left(\mathbf{x}, \mathbf{v}^{\prime \prime}\right) \mathbf{v}^{\prime \prime}+s \mathbf{v}^{\prime \prime}\right) d s}\right) .
$$


We can rewrite $\mathrm{H}_{2}$ into

$$
\frac{H_{2}\left(\mathbf{x}^{\prime \prime}+\tau_{-}\left(\mathbf{x}, \mathbf{v}^{\prime \prime}\right) \mathbf{v}^{\prime \prime}\right)}{\mathfrak{g}\left(\mathbf{v}^{\prime \prime}\right) \Upsilon\left(\mathbf{x}^{\prime \prime}+\tau_{-}\left(\mathbf{x}, \mathbf{v}^{\prime \prime}\right) \mathbf{v}^{\prime \prime}\right)}=\sigma_{a}\left(\mathbf{x}^{\prime \prime}+\tau_{-}\left(\mathbf{x}, \mathbf{v}^{\prime \prime}\right) \mathbf{v}^{\prime \prime}\right) e^{-\int_{0}^{\tau_{-}\left(\mathbf{x}, \mathbf{v}^{\prime \prime}\right)}} \sigma_{a}\left(\mathbf{x}^{\prime \prime}+s \mathbf{v}^{\prime \prime}\right) d s .
$$

Taking the ratio of (2.17) and (2.13), we obtain, after taking logarithm on both sides,

$$
\begin{aligned}
& \ln \left(\frac{H_{2}\left(\mathbf{x}^{\prime \prime}+\tau_{-}\left(\mathbf{x}, \mathbf{v}^{\prime \prime}\right) \mathbf{v}^{\prime \prime}\right) \mathfrak{g}\left(\mathbf{v}^{\prime}\right) \Upsilon\left(\mathbf{x}^{\prime}+\tau_{-}\left(\mathbf{x}, \mathbf{v}^{\prime}\right) \mathbf{v}^{\prime}\right)}{H_{1}\left(\mathbf{x}^{\prime}+\tau_{-}\left(\mathbf{x}, \mathbf{v}^{\prime}\right) \mathbf{v}^{\prime}\right) \mathfrak{g}\left(\mathbf{v}^{\prime \prime}\right) \Upsilon\left(\mathbf{x}^{\prime \prime}+\tau_{-}\left(\mathbf{x}, \mathbf{v}^{\prime \prime}\right) \mathbf{v}^{\prime \prime}\right)}\right) \\
= & \int_{0}^{\tau_{-}\left(\mathbf{x}, \mathbf{v}^{\prime}\right)} \sigma_{a}\left(\mathbf{x}^{\prime}+s \mathbf{v}^{\prime}\right) d s-\int_{0}^{\tau_{-}\left(\mathbf{x}, \mathbf{v}^{\prime \prime}\right)} \sigma_{a}\left(\mathbf{x}^{\prime \prime}+s \mathbf{v}^{\prime \prime}\right) d s .
\end{aligned}
$$

We can now differentiate this result with respect to $\tau_{-}\left(\mathbf{x}, \mathbf{v}^{\prime}\right)$ (which is equivalent to the directional differentiation $\mathbf{v}^{\prime} \cdot \nabla_{\mathbf{x}}$ ) and use relation (2.16) to obtain the quantity $\left(1-\frac{\mathbf{v}^{\prime} \cdot \overline{\mathbf{v}}}{\mathbf{v}^{\prime \prime} \cdot \overline{\mathbf{v}}}\right) \sigma_{a}(\mathbf{x})$. Note that for any point $\mathbf{x} \in \Omega$ located on the line determined by $\mathbf{x}^{\prime}$ and $\mathbf{x}^{\prime \prime}, \frac{\mathbf{v}^{\prime} \cdot \overline{\mathbf{v}}}{\mathbf{v}^{\prime \prime} \cdot \overline{\mathbf{v}}}=-1$ (because $\mathbf{x}$ lies between $\mathbf{x}^{\prime}$ and $\mathbf{x}^{\prime \prime}$ ). The result in this case reduces to that in the case of two collimated sources: $\left(1-\frac{\mathbf{v}^{\prime} \cdot \overline{\mathbf{v}}}{\mathbf{v}^{\prime \prime} \cdot \overline{\mathbf{v}}}\right) \sigma_{a}(\mathbf{x})=2 \sigma_{a}(\mathbf{x})$.

2.3. Reconstruction with cone-limited sources. The reconstruction procedures in the previous sections work only when the illumination function $g$ takes the required special forms. For more general sources, we do not have similar explicit reconstruction procedures. In fact, it is not clear whether or not data from an arbitrary source would uniquely determine the absorption coefficient. We consider here a reconstruction method for a source that is slightly more general than the sources used in sections 2.1 and 2.2 but which still possess certain causality properties of those sources.

Let $\mathbf{v}^{0}$ be a selected direction pointing inside the domain $\Omega$. We intend to construct a source such that the transport equation (2.1) with the source is causal along direction $\mathbf{v}^{0}$. Such causality would allow us to derive a direct layer peeling method that solves the inverse problem in one pass in a non-iterative manner. We require the source $g(\mathbf{x}, \mathbf{v})$ satisfy the following condition:

$$
g(\mathbf{x}, \mathbf{v})=0 \quad \text { for any } \quad \mathbf{v} \notin V\left(\mathbf{v}^{0}, \theta^{0}\right)=\left\{\mathbf{v} \in \mathbb{S}^{d-1} \mid \mathbf{v} \cdot \mathbf{v}^{0} \geq \cos \theta^{0}\right\},
$$

where the cone $V\left(\mathbf{v}^{0}, \theta^{0}\right)$ is determined by the selected direction $\mathbf{v}^{0} \in \mathbb{S}^{d-1}$ and the half-aperture $0<\theta^{0}<\pi / 2$. A practically important case that satisfies the assumption (2.19) is when the physical source can be separated from the object occupying the domain $\Omega$ by a hyper-plane. This limits the angle at which $\Omega$ is visible from the source, thus the rays entering $\Omega$ are limited to some cone of directions $V\left(\mathbf{v}^{0}, \theta^{0}\right)$ with $\mathbf{v}^{0}$ orthogonal to the separating hyper-plane. Then the resulting effective boundary conditions satisfy (2.19). In fact, in such a case we can extend $\Omega$ to a $d$-dimensional hyper-cube $\tilde{\Omega}$ such that $g(\mathbf{x}, \mathbf{v})$ is non-zero only on one side of the cube which is orthogonal to $\mathbf{v}^{0}$, as shown in figure 2.2 .

To simplify the presentation of the method we consider here the case $d=2$, although the algorithm remains essentially the same for $d=3$. We assume that $\tilde{\Omega}$ is the square $[0, L]^{2}$ with $\sigma_{a}(\mathbf{x})=0$ for $\mathbf{x} \in \tilde{\Omega} \backslash \Omega$. We also assume that the rays enter the domain from the bottom side $\{(x, y) \mid x \in(0, L), y=0\}$, so $\mathbf{v}^{0}=(0,1)$. The source $g(\mathbf{x}, \mathbf{v})$ is only non-zero on the bottom side. Thus the equation (2.1) is causal in the 


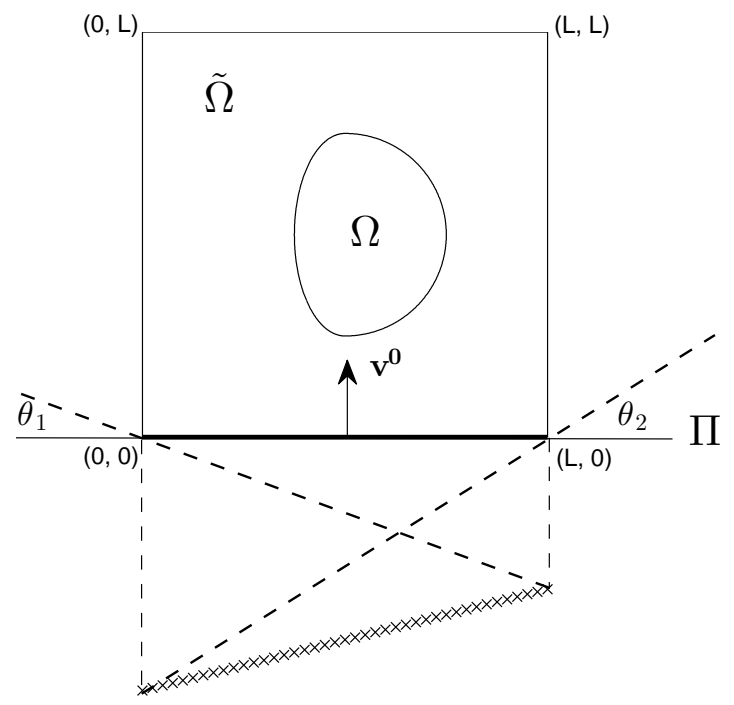

FIG. 2.2. Domain $\Omega$ and its extension to a hyper-cube $\tilde{\Omega}$. The physical sources located on a line marked with $\times$ are separated from $\Omega$ by a hyper-plane $\Pi$. The boundary condition is non-zero only on the bottom side of $\tilde{\Omega}$ (thick black part) that is orthogonal to $\mathbf{v}^{0}$. The half-aperture $\theta^{0}$ of the cone $V\left(\mathbf{v}^{0}, \theta^{0}\right)$ is given by $\theta^{0}=\pi / 2-\min \left\{\theta_{1}, \theta_{2}\right\}$.

$y$ direction; see figure 2.2 for the details of the setup. We use the parametrization $\mathbf{v}=(\cos \theta, \sin \theta)$ so that $\mathbf{v} \in V\left(\mathbf{v}^{0}, \theta^{0}\right)$ becomes $\theta \in\left[\pi / 2-\theta^{0}, \pi / 2+\theta^{0}\right]$. This implies that we can divide $(2.1)$ by $\sin \theta(>0)$, so that the free transport equation together with its boundary condition can be written as

$$
\begin{aligned}
u_{y}(x, y, \theta) & =-\cot \theta u_{x}(x, y, \theta)-\csc \theta \sigma_{a}(x, y) u(x, y, \theta), \\
u(x, 0, \theta) & =g(x, \theta), \quad x \in(0, L), \theta \in\left[\frac{\pi}{2}-\theta^{0}, \frac{\pi}{2}+\theta^{0}\right], \\
u(0, y, \theta) & =0, \quad y \in(0, L), \theta \in\left[\frac{\pi}{2}-\theta^{0}, \frac{\pi}{2}\right), \\
u(L, y, \theta) & =0, \quad y \in(0, L), \theta \in\left(\frac{\pi}{2}, \frac{\pi}{2}+\theta^{0}\right],
\end{aligned}
$$

where we observe that no boundary condition is needed on the top side of the domain $\tilde{\Omega},\{(x, y) \mid x \in(0, L), y=L\}$. The interior data $H(x, y)$ now takes the form

$$
H(x, y)=\Upsilon(x, y) \sigma_{a}(x, y) \int_{\pi / 2-\theta^{0}}^{\pi / 2+\theta^{0}} u(x, y, \theta) d \theta \equiv \Upsilon(x, y) \sigma_{a}(x, y)\langle u(x, y, \theta)\rangle_{\theta^{0}} .
$$

The role of the temporal variable in (2.20) is played by $y$ (the system is causal in $y$ ), so we can apply a first order time stepping procedure to obtain a semi-discrete inversion scheme. Let us discretize (2.20) in $y$ on a grid with nodes $y_{j}, j=0, \ldots, N_{y}$, where $y_{0}=0, y_{N_{y}}=L$, and the grid steps are $h_{k}=y_{k}-y_{k-1}, k=1, \ldots, N_{y}$. For the semi-discrete quantities we use notation

$$
u^{(j)}(x, \theta) \approx u\left(x, y_{j}, \theta\right), \quad \sigma_{a}^{(j)}(x) \approx \sigma_{a}\left(x, y_{j}\right), \quad j=0, \ldots, N_{y} .
$$

To reconstruct $\sigma_{a}$ with $\Upsilon$ known, we apply a forward Euler time stepping scheme to (2.20) to obtain the following explicit reconstruction procedure. 
1. Initialize $u^{(0)}(x, \theta)=g(x, \theta)$, and

$$
\sigma_{a}^{(0)}(x)=\frac{H(x, 0)}{\Upsilon(x, 0)\langle g(x, \theta)\rangle_{\theta^{0}}}, \quad x \in[0, L] .
$$

2. For $j=1, \ldots, N_{y}$, compute

$$
u^{(j)}(x, \theta)=\left(\mathcal{I}-h_{j} \cot \theta \partial_{x}-h_{j} \csc \theta \sigma_{a}^{(j-1)}(x)\right) u^{(j-1)}(x, \theta)
$$

for $x \in(0, L)$ and $\theta \in\left[\pi / 2-\theta^{0}, \pi / 2+\theta^{0}\right]$. At the boundary $x \in\{0, L\}$ set

$$
\begin{aligned}
u^{(j)}(0, \theta) & =0, \quad \theta \in\left[\frac{\pi}{2}-\theta^{0}, \frac{\pi}{2}\right), \\
u^{(j)}(L, \theta) & =0, \quad \theta \in\left(\frac{\pi}{2}, \frac{\pi}{2}+\theta^{0}\right],
\end{aligned}
$$

and compute the reconstruction

$$
\sigma_{a}^{(j)}(x)=\frac{H\left(x, y_{j}\right)}{\Upsilon\left(x, y_{j}\right)\left\langle u^{(j)}(x, \theta)\right\rangle_{\theta^{0}}}, \quad x \in[0, L] .
$$

The above method is quite stable in practice as demonstrated in the numerical examples in Section 5.1. However, the discretization of $\partial_{x}$ in (2.22) should be compatible with the grid refinement in $y$ to retain stability of the forward Euler scheme. If a coarser discretization in $y$ compared to that in $x$ is desired, then (2.22) can be replaced by a semi-implicit scheme

$$
\left(\mathcal{I}+h_{j} \cot \theta \partial_{x}\right) u^{(j)}(x, \theta)=\left(\mathcal{I}-h_{j} \csc \theta \sigma_{a}^{(j-1)}(x)\right) u^{(j-1)}(x, \theta),
$$

which requires solving a first order differential (difference if discretized) equation in $x$ at every step $j$ with boundary conditions (2.23). Generalizing this method to reconstruct simultaneously two coefficients $\Upsilon$ and $\sigma_{a}$ remains a topic of future study.

We conclude Section 2 by summarizing the reconstruction results for nonscattering media that we have introduced into the following uniqueness and stability theorem.

TheOREM 2.2. Let $\left(\Upsilon, \sigma_{a}\right)$ and $\left(\tilde{\Upsilon}, \tilde{\sigma}_{a}\right)$ be two sets of coefficients in (1.3) and (2.1) satisfying the assumptions in (A1), and $g_{1}(\mathbf{x}, \mathbf{v})$ and $g_{2}(\mathbf{x}, \mathbf{v})$ be two source functions of the form (2.4) or (2.12). Let $\left(H_{1}, H_{2}\right)$ and $\left(\tilde{H}_{1}, \tilde{H}_{2}\right)$ be the corresponding interior data. Then (a) $H_{1}=\tilde{H}_{1}$ implies $\sigma_{a}(\mathbf{x})=\tilde{\sigma}_{a}(\mathbf{x})$ if $\Upsilon=\Upsilon$; ; and (b) $\left(H_{1}, H_{2}\right)=\left(\tilde{H}_{1}, \tilde{H}_{2}\right)$ implies $\left(\Upsilon, \sigma_{a}\right)=\left(\tilde{\Upsilon}, \tilde{\sigma}_{a}\right)$. Moreover, the following stability result holds:

$$
\begin{aligned}
& \| \Upsilon(\mathbf{x}) \sigma_{a}(\mathbf{x}) e^{-\int_{0}^{\tau_{-}\left(\mathbf{x}, \mathbf{v}^{\prime}\right)} \sigma_{a}\left(\mathbf{x}-\tau_{-}\left(\mathbf{x}, \mathbf{v}^{\prime}\right) \mathbf{v}^{\prime}+s \mathbf{v}^{\prime}\right) d s} \\
& \quad-\tilde{\Upsilon}(\mathbf{x}) \tilde{\sigma}_{a}(\mathbf{x}) e^{-\int_{0}^{\tau_{-}\left(\mathbf{x}, \mathbf{v}^{\prime}\right)} \tilde{\sigma}_{a}\left(\mathbf{x}-\tau_{-}\left(\mathbf{x}, \mathbf{v}^{\prime}\right) \mathbf{v}^{\prime}+s \mathbf{v}^{\prime}\right) d s} \|_{L^{\infty}(\Omega)} \\
& \leq C_{l}\left\|H_{l}-\tilde{H}_{l}\right\|_{L^{\infty}(\Omega)}, \quad l=1,2
\end{aligned}
$$

$C_{l}$ being a constant that depends on $g_{l}$ but is independent of the data $H_{l}$ and $\tilde{H}_{l}$.

\section{QPAT of scattering media}

We now consider the QPAT problem for scattering media. When the scattering effect is very weak, the results obtained in the previous section could be used to obtain a good approximation of the reconstruction. We thus assume that the scattering 
effects are sufficiently strong so that neglecting them would deteriorate significantly the quality of the reconstructions. On the other hand, we assume that the scattering effect is not strong enough for us to model the light propagation process with the diffusion model, for which explicit reconstruction strategies have been proposed [14, $16,18]$.

3.1. Stability and uniqueness results. Unlike the non-scattering regime where we have unique and stable reconstruction using only a small number of interior data sets, in scattering media, we have only stability and uniqueness results from information of the full albedo operator:

$$
\mathcal{A}: \begin{array}{ll}
L^{1}\left(\Gamma_{-}, d \boldsymbol{\xi}\right) & \rightarrow L^{1}(\Omega), \\
g(\mathbf{x}, \mathbf{v}) & \mapsto \mathcal{A} g=H(\mathbf{x}) .
\end{array}
$$

It is the clear that $\mathcal{A}$ depends on all three coefficients $\Upsilon, \sigma_{a}$, and $\sigma_{s}$. We denote by $\|\mathcal{A}\|_{\mathcal{L}\left(L^{1}\left(\Gamma_{-}, d \boldsymbol{\xi}\right) ; L^{1}(\Omega)\right)}$ the norm of $\mathcal{A}$ as a linear operator from $L^{1}\left(\Gamma_{-}, d \boldsymbol{\xi}\right)$ to $L^{1}(\Omega)$.

We then have the following stability estimate following the analysis in [13, Theorem 2.3].

THEOREM 3.1. Let $\left(\Upsilon, \sigma_{a}, \sigma_{s}\right)$ and $\left(\tilde{\Upsilon}, \tilde{\sigma}_{a}, \tilde{\sigma}_{s}\right)$ be two set of coefficients satisfying the regularity assumptions in $(\mathbf{A} 1)$, and $\mathcal{A}, \tilde{\mathcal{A}}$ the corresponding albedo operators. Denote $\sigma=\sigma_{a}+\sigma_{s}$ and $\tilde{\sigma}=\tilde{\sigma}_{a}+\tilde{\sigma}_{s}$. Then the following result holds:

$$
\begin{aligned}
& \int_{0}^{\tau_{+}\left(\mathbf{x}^{\prime}, \mathbf{v}^{\prime}\right)} \mid \Upsilon\left(\mathbf{x}^{\prime}+t \mathbf{v}^{\prime}\right) \sigma_{a}\left(\mathbf{x}^{\prime}+t \mathbf{v}^{\prime}\right) e^{-\int_{0}^{t} \sigma\left(\mathbf{x}^{\prime}+s \mathbf{v}^{\prime}\right) d s} \\
& -\tilde{\Upsilon}\left(\mathbf{x}^{\prime}+t \mathbf{v}^{\prime}\right) \tilde{\sigma}_{a}\left(\mathbf{x}^{\prime}+t \mathbf{v}^{\prime}\right) e^{-\int_{0}^{t} \tilde{\sigma}\left(\mathbf{x}^{\prime}+s \mathbf{v}^{\prime}\right) d s} \mid d t \\
\leq & \|\mathcal{A}-\tilde{\mathcal{A}}\|_{\mathcal{L}\left(L^{1}\left(\Gamma_{-}, d \boldsymbol{\xi}\right) ; L^{1}(\Omega)\right)}
\end{aligned}
$$

for any point $\left(\mathbf{x}^{\prime}, \mathbf{v}^{\prime}\right) \in \Gamma_{-}$.

The result is a slight modification of the result in [13] where the singular part of the Schwartz kernel of the albedo operator is analyzed in detail. The only change that we have to make is to include the Grüneisen coefficient $\Upsilon$ in the final step of the analysis. We thus will not repeat the lengthy analysis here, but instead refer to [13].

It turns out that the stability result (3.2) leads to the unique reconstruction of the coefficients in some simplified situations. More precisely, if one of the three coefficients is known, we can reconstruct the other two coefficients uniquely as summarized in the following corollary.

COROLlaRY 3.2. Let $\left(\Upsilon, \sigma_{a}, \sigma_{s}\right)$ and $\left(\tilde{\Upsilon}, \tilde{\sigma}_{a}, \tilde{\sigma}_{s}\right)$ be two set of coefficients satisfying the regularity assumptions in (A1). Then the following statements hold: (a) If $\Upsilon=\tilde{\Upsilon}$, then $\mathcal{A}=\tilde{\mathcal{A}}$ implies $\left(\sigma_{a}, \sigma_{s}\right)=\left(\tilde{\sigma}_{a}, \tilde{\sigma}_{s}\right) ;$ (b) If $\sigma_{s}=\tilde{\sigma}_{s}$, then $\mathcal{A}=\tilde{\mathcal{A}}$ implies $\left(\Upsilon, \sigma_{a}\right)=$ $\left(\tilde{\Upsilon}, \tilde{\sigma}_{a}\right)$; (c) If $\sigma_{a}=\tilde{\sigma}_{a}$, then $\mathcal{A}=\tilde{\mathcal{A}}$ implies $\left(\Upsilon, \sigma_{s}\right)=\left(\tilde{\Upsilon}, \tilde{\sigma}_{s}\right)$.

Proof. Theorem 3.1 implies that we can reconstruct uniquely

$$
\mathcal{M}\left(\mathbf{x}^{\prime}, \mathbf{v}^{\prime}, t\right)=\Upsilon\left(\mathbf{x}^{\prime}+t \mathbf{v}^{\prime}\right) \sigma_{a}\left(\mathbf{x}^{\prime}+t \mathbf{v}^{\prime}\right) e^{-\int_{0}^{t} \sigma\left(\mathbf{x}^{\prime}+s \mathbf{v}^{\prime}\right) d s}, \quad\left(\mathbf{x}^{\prime}, \mathbf{v}^{\prime}\right) \in \Gamma_{-} .
$$

We can reconstruct the same quantity for a different point on $\left(\mathbf{x}^{\prime}+\tau_{+}\left(\mathbf{x}^{\prime}, \mathbf{v}^{\prime}\right) \mathbf{v}^{\prime},-\mathbf{v}^{\prime}\right)$ on $\Gamma_{-}$with $t$ replaced by $\tau_{+}\left(\mathbf{x}^{\prime}, \mathbf{v}^{\prime}\right)-t: \mathcal{M}\left(\mathbf{x}^{\prime}+\tau_{+}\left(\mathbf{x}^{\prime}, \mathbf{v}^{\prime}\right) \mathbf{v}^{\prime},-\mathbf{v}^{\prime}, \tau_{+}\left(\mathbf{x}^{\prime}, \mathbf{v}^{\prime}\right)-t\right)$. Taking the $\log$ of the ratio of the two quantities, we obtain 


$$
\begin{aligned}
& \ln \frac{\mathcal{M}\left(\mathbf{x}^{\prime}+\tau_{+}\left(\mathbf{x}^{\prime}, \mathbf{v}^{\prime}\right) \mathbf{v}^{\prime},-\mathbf{v}^{\prime}, \tau_{+}\left(\mathbf{x}^{\prime}, \mathbf{v}^{\prime}\right)-t\right)}{\mathcal{M}\left(\mathbf{x}^{\prime}, \mathbf{v}^{\prime}, t\right)} \\
= & -\int_{0}^{\tau_{+}\left(\mathbf{x}^{\prime}, \mathbf{v}^{\prime}\right)-t} \sigma\left(\mathbf{x}^{\prime}+\tau_{+}\left(\mathbf{x}^{\prime}, \mathbf{v}^{\prime}\right)-s \mathbf{v}^{\prime}\right) d s+\int_{0}^{t} \sigma\left(\mathbf{x}^{\prime}+s \mathbf{v}^{\prime}\right) d s .
\end{aligned}
$$

Taking the derivative of the above quantity gives us $2 \sigma\left(\mathbf{x}^{\prime}+t \mathbf{v}^{\prime}\right)$ for a.e. $\left(\mathbf{x}^{\prime}, \mathbf{v}^{\prime}\right) \in$ $\Gamma_{-}$and $t>0$. This in turn gives us $\sigma(\mathbf{x})$ for a.e. $\mathbf{x} \in \Omega$. Once $\sigma(\mathbf{x})$ is uniquely determined, we reconstruct $\Upsilon \sigma_{a} \equiv \mu$ uniquely from $\mathcal{M}\left(\mathbf{x}^{\prime}, \mathbf{v}^{\prime}, t\right)$. (a) If $\Upsilon$ is known already, then $\sigma_{a}$ is known from $\mu$, and thus $\sigma-\sigma_{a}$ would give us $\sigma_{s}$; (b) If $\sigma_{s}$ is known, $\sigma-\sigma_{s}$ would give us $\sigma_{a}$. $\Upsilon$ is then known from $\mu$; (c) If $\sigma_{a}$ is known, then $\mu$ would give us $\Upsilon$ and $\sigma$ would give us $\sigma_{s}$. This completes the proof.

Remark 3.3. Case (a) was presented in [13]. The construction of $\mathcal{M}\left(\mathbf{x}^{\prime}, \mathbf{v}^{\prime}, t\right)$ and $\mathcal{M}\left(\mathbf{x}^{\prime}+\tau_{+}\left(\mathbf{x}^{\prime}, \mathbf{v}^{\prime}\right) \mathbf{v}^{\prime},-\mathbf{v}^{\prime}, \tau_{+}\left(\mathbf{x}^{\prime}, \mathbf{v}^{\prime}\right)-t\right)$ is in the same spirit as the construction of (2.5) and (2.10) (or (2.13) and (2.17)) using collimated (or point) sources in the previous section.

The above stability and uniqueness results, however, do not guarantee the unique reconstruction of all three coefficients simultaneously. In fact, theory based on the diffusion model in [14] states that one can not simultaneously reconstruct all three coefficient uniquely unless additional information is available [16]. Moreover, unlike the situation in the non-scattering regime, no explicit reconstruction method can be derived in the scattering media. We thus have to rely mainly on other computational methods for the reconstructions. We now derive a numerical reconstruction method based on the Born approximation of the nonlinear inverse problem.

3.2. Linearized reconstruction with Born approximation. We linearize around some known, not necessarily constant, background optical properties $\sigma_{a 0}(\mathbf{x})$ and $\sigma_{s 0}(\mathbf{x})$. To be more precise, we assume

$$
\sigma_{a}(\mathbf{x})=\sigma_{a 0}(\mathbf{x})+\tilde{\sigma}_{a}(\mathbf{x}), \quad \sigma_{s}(\mathbf{x})=\sigma_{s 0}(\mathbf{x})+\tilde{\sigma}_{s}(\mathbf{x}),
$$

where the perturbations are small in the sense that $\left\|\frac{\tilde{\sigma}_{a}(\mathbf{x})}{\sigma_{a 0}}\right\|_{L^{\infty}(\Omega)} \ll 1$ and $\left\|\frac{\tilde{\sigma}_{s}(\mathbf{x})}{\sigma_{s 0}}\right\|_{L^{\infty}(\Omega)} \ll 1$. Note again that $\Upsilon(\mathbf{x})$ is not assumed in perturbative form since the inverse problem of reconstructing $\Upsilon$ is linear (because the data $H$ is linearly proportional to the unknown $\Upsilon$ ).

In practice, the background properties can be obtained through a priori knowledge such as statistics of existing data. They can also be obtained by reconstructions using a less accurate, thus usually computationally cheaper, model of light propagation, for instance the diffusion approximation $[14,16]$.

The solution of the radiative transport problem with coefficients $\sigma_{a}$ and $\sigma_{s}$ can then be written as

$$
u(\mathbf{x}, \mathbf{v})=U(\mathbf{x}, \mathbf{v})+\tilde{u}(\mathbf{x}, \mathbf{v}),
$$

where $U(\mathbf{x}, \mathbf{v})$ is the solution of the transport equation (1.1) with the known background coefficients $\sigma_{a 0}$ and $\sigma_{s 0}$, and $\tilde{u}(\mathbf{x}, \mathbf{v})$ is the perturbation in the solution caused by the perturbations $\tilde{\sigma}_{a}(\mathbf{x})$ and $\tilde{\sigma}_{s}(\mathbf{x})$ in the coefficients. The equation satisfied by the perturbation $\tilde{u}(\mathbf{x}, \mathbf{v})$, to the first order, is

$$
\begin{aligned}
\mathbf{v} \cdot \nabla \tilde{u}(\mathbf{x}, \mathbf{v})+\sigma_{a 0}(\mathbf{x}) \tilde{u} & =\sigma_{s 0}(\mathbf{x}) K(\tilde{u})-\tilde{\sigma}_{a}(\mathbf{x}) U+\tilde{\sigma}_{s}(\mathbf{x}) K(U), & & \text { in } X, \\
\tilde{u}(\mathbf{x}, \mathbf{v}) & =0, & & \text { on } \Gamma_{-} .
\end{aligned}
$$


It is can be shown, using the fact that $u$ is Fréchet differentiable with respective to $\sigma_{a}$ and $\sigma_{s}[37,38,79]$, that the terms omitted are indeed high order terms.

We now introduce the (adjoint) Green's function $G(\mathbf{x}, \mathbf{v} ; \mathbf{y})$ for the transport problem with background optical properties, the solution of the following adjoint transport equation:

$$
\begin{aligned}
-\mathbf{v} \cdot \nabla G(\mathbf{x}, \mathbf{v} ; \mathbf{y})+\sigma_{a 0}(\mathbf{x}) G(\mathbf{x}, \mathbf{v} ; \mathbf{y}) & =\sigma_{s 0}(\mathbf{x}) K(G)(\mathbf{x}, \mathbf{v} ; \mathbf{y})-\delta(\mathbf{x}-\mathbf{y}), & & \text { in } X, \\
G(\mathbf{x}, \mathbf{v} ; \mathbf{y}) & =0, & & \text { on } \Gamma_{+},
\end{aligned}
$$

where $\delta$ is the usual Dirac delta function. Note that since the transport operator is not self-adjoint, the boundary condition is now put on $\Gamma_{+}$. Rigorously speaking, this equation only holds in the weak sense; we need to multiply it with a test function in $\mathcal{C}_{+}\left(\Omega \times \mathbb{S}^{d-1}\right)$ (that is the space of continuous positive functions from $\Omega \times \mathbb{S}^{d-1}$ to $\mathbb{R}$ ). This requires that the coefficients $\sigma_{a 0}$ and $\sigma_{s 0}$ are at least continuous in $\bar{\Omega}$.

We can now multiply (3.7) by $\Upsilon G$ and integrate over $X$, and multiply (3.8) by $\Upsilon \tilde{u}$ and integrate over $X$, and subtract the results to show that

$$
\begin{aligned}
\Upsilon(\mathbf{x})\langle\tilde{u}(\mathbf{x}, \mathbf{v})\rangle_{\mathbf{v}}=\int_{\Omega} \Upsilon(\mathbf{y}) \tilde{\sigma}_{a}(\mathbf{y})\langle & U(\mathbf{y}, \mathbf{v}) G(\mathbf{y}, \mathbf{v} ; \mathbf{x})\rangle_{\mathbf{v}} d \mathbf{y} \\
& \quad-\int_{\Omega} \Upsilon(\mathbf{y}) \tilde{\sigma}_{s}(\mathbf{y})\langle K(U)(\mathbf{y}, \mathbf{v}) G(\mathbf{y}, \mathbf{v} ; \mathbf{x})\rangle_{\mathbf{v}} d \mathbf{y} .
\end{aligned}
$$

The perturbations in (3.5) and (3.6) imply that the interior data $H$ is now given, to the first order, by

$$
H=\Upsilon_{a 0}\langle U\rangle_{\mathbf{v}}+\Upsilon \tilde{\sigma}_{a}\langle U\rangle_{\mathbf{v}}+\Upsilon \sigma_{a 0}\langle\tilde{u}\rangle_{\mathbf{v}}
$$

Combining (3.10) and (3.9), we can show that

$$
\frac{H}{\sigma_{a 0}\langle U\rangle_{\mathbf{v}}}(\mathbf{x})=\mathcal{I}(\Upsilon)+\mathcal{L}^{a}\left(\Upsilon \tilde{\sigma}_{a}\right)+\mathcal{L}^{s}\left(\Upsilon \tilde{\sigma}_{s}\right)
$$

where $\mathcal{I}$ is the identity operator and the operators $\mathcal{L}^{a}$ and $\mathcal{L}^{s}$ are defined, respectively, as

$$
\begin{aligned}
& \mathcal{L}^{a}\left(\Upsilon \tilde{\sigma}_{a}\right)=\frac{\Upsilon \tilde{\sigma}_{a}}{\sigma_{a 0}}+\int_{\Omega} \Upsilon(\mathbf{y}) \tilde{\sigma}_{a}(\mathbf{y}) \frac{\langle U G\rangle_{\mathbf{v}}}{\langle U\rangle_{\mathbf{v}}}(\mathbf{y} ; \mathbf{x}) d \mathbf{y}, \\
& \mathcal{L}^{s}\left(\Upsilon \tilde{\sigma}_{s}\right)=-\int_{\Omega} \Upsilon(\mathbf{y}) \tilde{\sigma}_{s}(\mathbf{y}) \frac{\langle K(U) G\rangle_{\mathbf{v}}}{\langle U\rangle_{\mathbf{v}}}(\mathbf{y} ; \mathbf{x}) d \mathbf{y} .
\end{aligned}
$$

Here we have normalized the data by $\sigma_{a 0}\langle U\rangle_{\mathbf{v}}$. This can be done when $\langle U\rangle_{\mathbf{v}}(\mathbf{x}) \neq 0$, which can be guaranteed by selecting an appropriate illumination source $g(\mathbf{x}, \mathbf{v})$. The normalization makes the sizes of the kernels of the integral operators $\mathcal{L}^{a}$ and $\mathcal{L}^{s}$ on the same order at all locations. This is important due to the fact that the strength of optical signals usually varies over several orders of magnitude across the domain of interest. The normalization results in well-balanced matrix elements when the integral equation (3.11) is discretized for numerical solution.

Equation (3.11) is a linear integral equation for the three variables $\Upsilon$, $\Upsilon \tilde{\sigma}_{a}$, and $\Upsilon \tilde{\sigma}_{s}$. The kernels for the operator $\mathcal{L}^{a}$ and those for $\mathcal{L}^{s},\langle U G\rangle_{\mathbf{v}} /\langle U\rangle_{\mathbf{v}}$, and $\langle K(U) G\rangle_{\mathbf{v}} /\langle U\rangle_{\mathbf{v}}$ are known since they only involve the solutions of the forward and adjoint transport equations with background optical properties $\sigma_{a 0}$ and $\sigma_{s 0}$. It remains to solve (3.11) to reconstruct the unknowns $\left(\Upsilon, \Upsilon \tilde{\sigma}_{a}\right.$, and $\left.\Upsilon \tilde{\sigma}_{s}\right)$, and then the real coefficients $\left(\Upsilon, \tilde{\sigma}_{a}\right.$, and $\left.\tilde{\sigma}_{s}\right)$. 
In practice, we do not have the full albedo data, but only a finite number of data sets generated from different sources. Let us assume that there are $N_{g}$ data sets collected from $N_{g}$ illuminations. The data sets are denoted by $\left\{H_{i}\right\}_{i=1}^{N_{g}}$ and the corresponding illuminations are denoted by $\left\{g_{i}\right\}_{i=1}^{N_{g}}$. The system of linear integral equation with $N_{g}$ data sets can be written as

$$
\mathcal{L}\left(\begin{array}{c}
\Upsilon \\
\Upsilon \tilde{\sigma}_{a} \\
\Upsilon \tilde{\sigma}_{s}
\end{array}\right) \equiv\left(\begin{array}{ccc}
\mathcal{I} & \mathcal{L}_{1}^{a} & \mathcal{L}_{1}^{s} \\
\vdots & \vdots & \vdots \\
\mathcal{I} & \mathcal{L}_{i}^{a} & \mathcal{L}_{i}^{s} \\
\vdots & \vdots & \vdots \\
\mathcal{I} & \mathcal{L}_{N_{g}}^{a} & \mathcal{L}_{N_{g}}^{s}
\end{array}\right)\left(\begin{array}{c}
\Upsilon \\
\Upsilon \tilde{\sigma}_{a} \\
\Upsilon \tilde{\sigma}_{s}
\end{array}\right)=\frac{1}{\sigma_{a 0}}\left(\begin{array}{c}
H_{1} /\left\langle U_{1}\right\rangle_{\mathbf{v}} \\
\vdots \\
H_{i} /\left\langle U_{i}\right\rangle_{\mathbf{v}} \\
\vdots \\
H_{N_{g}} /\left\langle U_{N_{g}}\right\rangle_{\mathbf{v}}
\end{array}\right) \equiv\left(\begin{array}{c}
h_{1} \\
\vdots \\
h_{i} \\
\vdots \\
h_{N_{g}}
\end{array}\right)
$$

where $\mathcal{L}_{i}^{a}$ and $\mathcal{L}_{i}^{s}$ are the same operators defined in (3.12) and (3.13), respectively, with $U$ replaced with $U_{i}$.

The integral formulation can be discretized to get a linear system of equations. Let us assume that we have a numerical procedure, say a quadrature rule, to discretize the integral equation (3.9), and assume that we discretize $\tilde{\sigma}_{a}(\mathbf{x})$ on a mesh of $N_{\Omega}$ nodes, $\left\{\mathbf{y}_{k}\right\}_{k=1}^{N_{\Omega}}$. Then $\mathcal{L}$ will be matrix consisting of $N_{g} \times 3$ blocks, each having size $N_{\Omega} \times N_{\Omega}$. The $l k$ elements of the matrices are given by

$$
\left(\mathcal{L}_{i}^{a}\right)_{l k}=\frac{\delta_{l k}}{\sigma_{a 0}\left(\mathbf{x}_{l}\right)}+\xi_{k} \frac{\left\langle U_{i}\left(\mathbf{y}_{k}, \mathbf{v}\right) G\left(\mathbf{y}_{k}, \mathbf{v} ; \mathbf{x}_{l}\right)\right\rangle_{\mathbf{v}}}{\left\langle U_{i}\left(\mathbf{x}_{l}, \mathbf{v}\right)\right\rangle_{\mathbf{v}}}, \quad\left(\mathcal{L}_{i}^{s}\right)_{l k}=-\xi_{k} \frac{\left\langle K\left(U_{i}\right)\left(\mathbf{y}_{k}, \mathbf{v}\right) G\left(\mathbf{y}_{k}, \mathbf{v} ; \mathbf{x}_{l}\right)\right\rangle_{\mathbf{v}}}{\left\langle U_{i}\left(\mathbf{x}_{l}, \mathbf{v}\right)\right\rangle_{\mathbf{v}}},
$$

where $\xi_{k}\left(1 \leq k \leq N_{\Omega}\right)$ is the weight of the quadrature on the $k$-th element. For simplicity of notation, we will not differentiate from now on integral operators and their discrete equivalences, the matrices.

The system (3.14) can be over- or under-determined and so is often solved in the regularized least-square sense. More specifically, we solving the problem by solve the following minimization problem:

$$
\min _{\Upsilon, \Upsilon \tilde{\sigma}_{a}, \Upsilon \tilde{\sigma}_{s}}\left\|\mathcal{L}\left(\begin{array}{c}
\Upsilon \\
\Upsilon \tilde{\sigma}_{a} \\
\Upsilon \tilde{\sigma}_{s}
\end{array}\right)-\left(\begin{array}{c}
h_{1} \\
\vdots \\
h_{N_{g}}
\end{array}\right)\right\|_{l^{2}}^{2}+\rho\left\|\mathcal{D}\left(\begin{array}{c}
\Upsilon \\
\Upsilon \tilde{\sigma}_{a} \\
\Upsilon \tilde{\sigma}_{s}
\end{array}\right)\right\|_{l^{2}}^{2}
$$

where the first term is the data fidelity term and the second term is a Tikhonov regularization term with the strength of regularization given by $\rho$. The operator $\mathcal{D}$ is the discrete differentiation. The regularization term is needed due to the presence of noise in practice even though the inverse problem here is very stable compared to similar inverse transport problems in diffuse optical tomography $[9,11,77]$.

REMARK 3.4. The $l^{2}$ least squares formulation and Tikhonov regularization strategy (3.16) are adopted here mainly for their computational efficiency. One can employ different data fidelity and regularization terms such as those based on total variation (TV) and $l^{1}$ norms $[45,46]$. Different selection can be effective for different problems. We refer the interested reader to $[45,46]$ for detailed numerical analysis and comparison of performances of different approaches. The primary focus here is the properties of the inverse transport problems in QPAT, not the details of the numerical implementation. 
It is important to notice that the norm of the operator $\mathcal{L}^{s}$ is in general small compared to that of $\mathcal{L}^{a}$ and the identity operator $\mathcal{I}$, especially when the transport process is diffusive and the scattering is very isotropic. In those cases, $U(\mathbf{x}, \mathbf{v})$ is roughly independent of $\mathbf{v}$, so that $\|K(U)\|_{L^{1}(X)} \ll 1$. Thus $\langle K(U) G\rangle_{\mathbf{v}}$ is very small. In this case, when noise is present in the measured data, the reconstruction of the scattering coefficient $\sigma_{s}$ is easily corrupted by noise. That was observed in the past in optical tomography $[9,10,77]$. We thus separate the reconstruction into the following two cases.

3.2.1. Reconstructing $\left(\Upsilon, \sigma_{a}\right)$. To reconstruct $\tilde{\sigma}_{a}$ and $\Upsilon$ assuming $\sigma_{s}$ is known, we solve the simplified least-squares problem

$$
\min _{\Upsilon, \Upsilon \tilde{\sigma}_{a}}\left\|\left(\begin{array}{cc}
\mathcal{I} & \mathcal{L}_{1}^{a} \\
\vdots & \vdots \\
\mathcal{I} & \mathcal{L}_{N_{g}}^{a}
\end{array}\right)\left(\begin{array}{c}
\Upsilon \\
\Upsilon \tilde{\sigma}_{a}
\end{array}\right)-\left(\begin{array}{c}
h_{1} \\
\vdots \\
h_{N_{g}}
\end{array}\right)\right\|_{l^{2}}^{2}+\rho\left\|\mathcal{D}\left(\begin{array}{c}
\Upsilon \\
\Upsilon \tilde{\sigma}_{a}
\end{array}\right)\right\|_{l^{2}}^{2}
$$

We recover from the solution the coefficients $\Upsilon$ and $\tilde{\sigma}_{a}$. The solution of the leastsquares problem is simply

$$
\left(\begin{array}{c}
\Upsilon \\
\Upsilon \tilde{\sigma}_{a}
\end{array}\right)=\left[\left(\begin{array}{cc}
N_{g} \mathcal{I} & \sum_{i=1}^{N_{g}} \mathcal{L}_{i}^{a} \\
\sum_{i=1}^{N_{g}} \mathcal{L}_{i}^{a *} & \sum_{i=1}^{N_{g}=1} \mathcal{L}_{i}^{a *} \mathcal{L}_{i}^{a}
\end{array}\right)+\rho \mathcal{D}^{*} \mathcal{D}\right]^{-1}\left(\begin{array}{ccc}
\mathcal{I} & \ldots & \mathcal{I} \\
\mathcal{L}_{1}^{a *} & \ldots & \mathcal{L}_{N_{g}}^{a *}
\end{array}\right)\left(\begin{array}{c}
h_{1} \\
\vdots \\
h_{N_{g}}
\end{array}\right)
$$

where the superscript $*$ is used to denote the adjoint of an operator, and $\mathcal{I}^{*}=\mathcal{I}$. The inversion of the regularized normal operator is achieved by a linear solver, not direct inversion.

3.2.2. Reconstructing $\left(\sigma_{a}, \sigma_{s}\right)$ or $\left(\Upsilon, \sigma_{s}\right)$. To reconstruct either $\left(\sigma_{a}\right.$, $\left.\sigma_{s}\right)$ or $\left(\Upsilon, \sigma_{s}\right)$, we need to deal with an unbalanced system of equations caused by the smallness of the scattering component $\mathcal{L}^{s}$. We consider here only the case of reconstructing $\left(\sigma_{a}, \sigma_{s}\right)$. To obtain a similar algorithm to reconstruct $\left(\Upsilon, \sigma_{s}\right)$, we only need to replace the operator $\mathcal{L}^{a}$ in the algorithm by $\mathcal{I}$. We solve the simplified least-squares problem

$$
\min _{\Upsilon \tilde{\sigma}_{a}, \Upsilon \tilde{\sigma}_{s}}\left\|\left(\begin{array}{cc}
\mathcal{L}_{1}^{a} & \mathcal{L}_{1}^{s} \\
\vdots & \vdots \\
\mathcal{L}_{N_{g}}^{a} & \mathcal{L}_{N_{g}}^{s}
\end{array}\right)\left(\begin{array}{c}
\Upsilon \tilde{\sigma}_{a} \\
\Upsilon \tilde{\sigma}_{s}
\end{array}\right)-\left(\begin{array}{c}
h_{1} \\
\vdots \\
h_{N_{g}}
\end{array}\right)\right\|_{l^{2}}^{2}+\rho\left\|\mathcal{D}\left(\begin{array}{c}
\Upsilon \tilde{\sigma}_{a} \\
\Upsilon \tilde{\sigma}_{s}
\end{array}\right)\right\|_{l^{2}}^{2} .
$$

The normal equation for this minimization problem is

$$
\left(\begin{array}{cc}
\sum_{i=1}^{N_{g}} \mathcal{L}_{i}^{a *} \mathcal{L}_{i}^{a}+\rho \mathcal{D}_{a}^{*} \mathcal{D}_{a} & \sum_{i=1}^{N_{g}} \mathcal{L}_{i}^{a *} \mathcal{L}_{i}^{s} \\
\sum_{i=1}^{N_{g}} \mathcal{L}_{i}^{s *} \mathcal{L}_{i}^{a} & \sum_{i=1}^{N_{g}} \mathcal{L}_{i}^{s *} \mathcal{L}_{i}^{s}+\rho \mathcal{D}_{s}^{*} \mathcal{D}_{s}
\end{array}\right)\left(\begin{array}{c}
\Upsilon \tilde{\sigma}_{a} \\
\Upsilon \tilde{\sigma}_{s}
\end{array}\right)=\left(\begin{array}{c}
\sum_{i=1}^{N_{g}} \mathcal{L}_{i}^{a *} h_{i} \\
\sum_{i=1}^{N_{g}} \mathcal{L}_{i}^{s *} h_{i}
\end{array}\right),
$$

where $\mathcal{D}_{a}$ and $\mathcal{D}_{s}$ are such that $\mathcal{D}=\operatorname{diag}\left(\mathcal{D}_{a}, \mathcal{D}_{s}\right)$. Instead of solving this normal equation directly to get a solution similar to (3.18), we perform Gaussian elimination of the system to obtain

$$
\left(\begin{array}{cc}
\sum_{i=1}^{N_{g}} \mathcal{L}_{i}^{a *} \mathcal{L}_{i}^{a}+\rho \mathcal{D}_{a}^{*} \mathcal{D}_{a} \sum_{i=1}^{N_{g}} \mathcal{L}_{i}^{a *} \mathcal{L}_{i}^{s} \\
0 & \mathcal{L}^{\text {red }}
\end{array}\right)\left(\begin{array}{c}
\Upsilon \tilde{\sigma}_{a} \\
\Upsilon \tilde{\sigma}_{s}
\end{array}\right)=\left(\begin{array}{c}
\sum_{i=1}^{N_{g}} \mathcal{L}_{i}^{a *} h_{i} \\
h^{\text {red }}
\end{array}\right)
$$


where

$$
\mathcal{L}^{r e d}=\sum_{i=1}^{N_{g}} \mathcal{L}_{i}^{s *} \mathcal{L}_{i}^{s}+\rho \mathcal{D}_{s}^{*} \mathcal{D}_{s}-\sum_{i=1}^{N_{g}} \mathcal{L}_{i}^{s *} \mathcal{L}_{i}^{a}\left(\sum_{i=1}^{N_{g}} \mathcal{L}_{i}^{a *} \mathcal{L}_{i}^{a}+\rho \mathcal{D}_{a}^{*} \mathcal{D}_{a}\right)^{-1} \sum_{i=1}^{N_{g}} \mathcal{L}_{i}^{a *} \mathcal{L}_{i}^{s}
$$

and

$$
h^{\text {red }}=\sum_{i=1}^{N_{g}} \mathcal{L}_{i}^{s *} h_{i}-\left[\sum_{i=1}^{N_{g}} \mathcal{L}_{i}^{s *} \mathcal{L}_{i}^{a}\left(\sum_{i=1}^{N_{g}} \mathcal{L}_{i}^{a *} \mathcal{L}_{i}^{a}+\rho \mathcal{D}_{a}^{*} \mathcal{D}_{a}\right)^{-1} \sum_{i=1}^{N_{g}} \mathcal{L}_{i}^{a *} \mathcal{L}_{i}^{s}\right] \sum_{i=1}^{N_{g}} \mathcal{L}_{i}^{a *} h_{i} .
$$

This motivates the following two-step reconstruction procedure.

Step I. We reconstruct the coefficient $\Upsilon \tilde{\sigma}_{s}$ by solving the reduced linear system

$$
\mathcal{L}^{\text {red }} \Upsilon \tilde{\sigma}_{s}=h^{\text {red }}
$$

In the construction of the operator $\mathcal{L}^{\text {red }}$, we need to apply the inverse of the operator $\sum_{i=1}^{N_{g}} \mathcal{L}_{i}^{a *} \mathcal{L}_{i}^{a}+\rho \mathcal{D}_{a}^{*} \mathcal{D}_{a}$. We construct this inverse as accurately as possible. For large problems, we avoid constructing the inverse operator directly. Instead, we construct it implicitly. More precisely, to apply the inverse operator to any object $z$ to get $\hat{z}=\left(\sum_{i=1}^{N_{g}} \mathcal{L}_{i}^{a *} \mathcal{L}_{i}^{a}+\rho \mathcal{D}_{a}^{*} \mathcal{D}_{a}\right)^{-1} z$, we solve the linear system $\left(\sum_{i=1}^{N_{g}} \mathcal{L}_{i}^{a *} \mathcal{L}_{i}^{a}+\rho \mathcal{D}_{a}^{*} \mathcal{D}_{a}\right) \hat{z}=$ $z$ to maximum accuracy.

Step II. Once the coefficient $\Upsilon \tilde{\sigma}_{s}$ is reconstructed, we can eliminate it from the system to obtain a linear system for the reconstruction of $\Upsilon \tilde{\sigma}_{a}:\left(\sum_{i=1}^{N_{g}} \mathcal{L}_{i}^{a *} \mathcal{L}_{i}^{a}+\right.$ $\left.\rho \mathcal{D}_{a}^{*} \mathcal{D}_{a}\right) \Upsilon \tilde{\sigma}_{a}=\sum_{i=1}^{N_{g}} \mathcal{L}_{i}^{a *} h_{i}-\left(\sum_{i=1}^{N_{g}} \mathcal{L}_{i}^{a *} \mathcal{L}_{i}^{s}\right) \Upsilon \tilde{\sigma}_{s}$

3.3. Minimization-based nonlinear reconstruction scheme. To solve the full nonlinear inverse problem, we reformulate the problem into a regularized leastsquares formulation. More precisely, we minimize the following objective functional:

$$
\Phi\left(\Upsilon, \sigma_{a}, \sigma_{s}\right) \equiv \frac{1}{2} \sum_{i=1}^{N_{g}}\left\|\Upsilon \sigma_{a}\left\langle u_{i}\right\rangle_{\mathbf{v}}-H_{i}^{*}\right\|_{L^{2}(\Omega)}^{2}
$$

where $u_{i}$ is the solution of the transport equation with the $i$ th illumination, i.e., $u_{i}$ solves

$$
\begin{aligned}
\mathbf{v} \cdot \nabla u_{i}(\mathbf{x}, \mathbf{v})+\sigma_{a}(\mathbf{x}) u_{i}(\mathbf{x}, \mathbf{v}) & =\sigma_{s}(\mathbf{x}) K\left(u_{i}\right)(\mathbf{x}, \mathbf{v}), & & \text { in } X \\
u_{i}(\mathbf{x}, \mathbf{v}) & =g_{i}(\mathbf{x}, \mathbf{v}), & & \text { on } \Gamma_{-} .
\end{aligned}
$$

The interior data collected for illumination $g_{i}$ is denoted by $H_{i}^{*}$.

It is known that the functional (3.23) is Fréchet differentiable with respect to the unknowns provided that the coefficients satisfy the assumptions in (A1); see for instance $[37,38,79]$. We can thus use gradient-based minimization techniques to solve this problem. To calculate the Fréchet derivatives of the objective functional, we use the method of adjoint equations [93]. Let us denote by $w_{i}$ the solution of the following adjoint transport equation:

$$
\begin{aligned}
-\mathbf{v} \cdot \nabla w_{i}(\mathbf{x}, \mathbf{v})+\sigma_{a}(\mathbf{x}) w_{i}(\mathbf{x}, \mathbf{v}) & =\sigma_{s}(\mathbf{x}) K\left(w_{i}\right)(\mathbf{x}, \mathbf{v})+\Upsilon \sigma_{a}\left(\Upsilon \sigma_{a}\left\langle u_{i}\right\rangle_{\mathbf{v}}-H_{i}^{*}\right), & & \text { in } X, \\
w_{i}(\mathbf{x}, \mathbf{v}) & =0, & & \text { on } \Gamma_{+} .
\end{aligned}
$$

It is then straightforward to follow the standard calculations in [37, 38, 79] to show that the Fréchet derivatives can be computed as follows. 
TheOREm 3.5. The Fréchet derivatives of $\Phi$ are given by

$$
\begin{aligned}
\left\langle\frac{\partial \Phi}{\partial \Upsilon}, \widehat{\Upsilon}\right\rangle & =\sum_{i=1}^{N_{g}}\left\langle\left(\Upsilon \sigma_{a}\left\langle u_{i}\right\rangle_{\mathbf{v}}-H_{i}^{*}\right) \sigma_{a}\left\langle u_{i}\right\rangle_{\mathbf{v}}, \widehat{\Upsilon}\right\rangle_{L^{2}(X)}, \\
\left\langle\frac{\partial \Phi}{\partial \sigma_{a}}, \widehat{\sigma_{a}}\right\rangle & =\sum_{i=1}^{N_{g}}\left\langle\left(\Upsilon \sigma_{a}\left\langle u_{i}\right\rangle_{\mathbf{v}}-H_{i}^{*}\right) \Upsilon\left\langle u_{i}\right\rangle_{\mathbf{v}}-\left\langle u_{i} w_{i}\right\rangle_{\mathbf{v}}, \widehat{\sigma_{a}}\right\rangle_{L^{2}(X)}, \\
\left\langle\frac{\partial \Phi}{\partial \sigma_{s}}, \widehat{\sigma_{s}}\right\rangle & =\sum_{i=1}^{N_{g}}\left\langle\left\langle K\left(u_{i}\right) w_{i}\right\rangle_{\mathbf{v}}, \widehat{\sigma_{s}}\right\rangle_{L^{2}(X)}
\end{aligned}
$$

where $\langle\cdot, \cdot\rangle_{L^{2}(X)}$ denotes the usual inner product in $L^{2}(X)$.

Note that due to the fact that the problem of reconstructing $\Upsilon$ is a linear inverse problem, the Fréchet derivative of the objective functional with respect to $\Upsilon$ is independent of the adjoint solutions $\left\{w_{i}\right\}_{i=1}^{N_{g}}$. If we know $\sigma_{a}$ and $\sigma_{s}$ but are interested in reconstructing $\Upsilon$, then we can simply solve for $\Upsilon$ such that $\left\langle\frac{\partial \Phi}{\partial \Upsilon}, \widehat{\Upsilon}\right\rangle=0$ for any test function $\widehat{\Upsilon}$. This in turn gives us $\Upsilon \sigma_{a}\left\langle u_{i}\right\rangle_{\mathbf{v}}-H_{i}^{*}=0,1 \leq i \leq N_{g}$, which holds for every point $\mathbf{x}$ in $\Omega$. The least-squares solution of this overdetermined system is $\Upsilon=\sum_{i=1}^{N_{g}}\left(\left\langle u_{i}\right\rangle_{\mathbf{v}} H_{i}^{*}\right) / \sum_{i=1}^{N_{g}}\left(\sigma_{a}\left\langle u_{i}\right\rangle_{\mathbf{v}}^{2}\right)$. Thus we need only to solve the $N_{g}$ transport equations and evaluate $\left\langle u_{i}\right\rangle_{\mathbf{v}}$ to reconstruct $\Upsilon$.

We use the limited memory version of the BFGS quasi-Newton method to solve the minimization problem. The details of the implementation are documented in [79, 77]. In our numerical experiments, we only attempt to reconstruct two of the three coefficients. We observe that the algorithm converges very fast, even from initial guesses that are relatively far from the true coefficients. This confirms the theory developed in the diffusive regime $[14,16,18]$, which is that the problem is very well-conditioned when only two coefficients are sought. In the numerical simulation, we can add a small amount of regularization to the problem when the noise in the data is significant. This is done by adding a Tikhonov functional term, such as $\frac{\rho}{2}\left\|\left(\Upsilon, \sigma_{a}\right)-\left(\bar{\Upsilon}, \bar{\sigma}_{a}\right)\right\|_{\left(\mathcal{H}^{1}(\Omega)\right)^{2}}^{2}$ in the reconstruction of $\left(\Upsilon, \sigma_{a}\right)$ where $\bar{\Upsilon}, \bar{\sigma}_{a}$, and $\bar{\sigma}_{s}$ are values obtained with a priori knowledge, in the objective functional (3.23).

\section{The multi-spectral QPAT}

In practical applications of PAT, light of different wavelengths can be used to probe the properties of the medium at those wavelengths $[25,26,27,30,33,57,66$, $74,76,83,84,94,103,105]$. This is the idea of multi-spectral QPAT.

Let us denote by $\Lambda$ the set of wavelengths that can be accessed in practice. The radiative transport equation in this case takes the form

$$
\begin{aligned}
\mathbf{v} \cdot \nabla u(\mathbf{x}, \mathbf{v}, \lambda)+\sigma_{a}(\mathbf{x}, \lambda) u(\mathbf{x}, \mathbf{v}, \lambda) & =\sigma_{s}(\mathbf{x}, \lambda) K(u)(\mathbf{x}, \mathbf{v}, \lambda), & & \text { in } X \times \Lambda, \\
u(\mathbf{x}, \mathbf{v}, \lambda) & =g(\mathbf{x}, \mathbf{v}, \lambda), & & \text { on } \Gamma_{-} \times \Lambda,
\end{aligned}
$$

where the coefficients $\sigma_{a}(\mathbf{x}, \lambda)$ and $\sigma_{s}(\mathbf{x}, \lambda)$ are now functions of the wavelength. The interior datum collected in this case is now also a function of the wavelength

$$
H(\mathbf{x}, \lambda)=\Upsilon(\mathbf{x}, \lambda) \int_{\mathbb{S}^{d-1}} \sigma_{a}(\mathbf{x}, \lambda) u(\mathbf{x}, \mathbf{v}, \lambda) d \mathbf{v} .
$$

Due to the fact that the equations for different wavelengths are decoupled, we could not expect to reconstruct unknowns at one wavelength from data collected at other wavelengths, unless other a priori information is supplied. 
If the medium is non-scattering, the results in Section 2 enable us to reconstruct $\Upsilon(\mathbf{x}, \lambda)$ and $\sigma_{a}(\mathbf{x}, \lambda)$ with two well-chosen illuminations $g_{1}(\mathbf{x}, \mathbf{v}, \lambda)$ and $g_{2}(\mathbf{x}, \mathbf{v}, \lambda)$. The illuminations can be either collimated sources of the form $\mathfrak{g}(\mathbf{x}, \lambda) \delta\left(\mathbf{v}-\mathbf{v}^{\prime}\right)$ or point sources in space of the form $\mathfrak{g}(\mathbf{v}, \lambda) \delta\left(\mathbf{x}-\mathbf{x}^{\prime}\right)$. We can simply perform wavelength by wavelength reconstructions using the methods developed in Section 2.

4.1. Uniqueness of reconstruction. The theory developed in [16] for the diffusion equation confirms that we can reconstruct all three coefficients $\Upsilon, \sigma_{a}$, and $\sigma_{s}$ simultaneously with multi-spectral interior data under practically reasonable assumptions on the coefficients. Following [16], we take the following standard model for the unknown coefficients:

$$
\sigma_{a}(\mathbf{x}, \lambda)=\sum_{k=1}^{K} \alpha_{k}(\lambda) \sigma_{a}^{k}(\mathbf{x}), \quad \sigma_{s}(\mathbf{x}, \lambda)=\beta(\lambda) \sigma_{s}(\mathbf{x}), \quad \Upsilon(\mathbf{x}, \lambda)=\gamma(\lambda) \Upsilon(\mathbf{x}),
$$

where the functions $\left\{\alpha_{k}(\lambda)\right\}_{k=1}^{K}, \beta(\lambda)$, and $\gamma(\lambda)$ are assumed to be known. In other words, all three coefficient functions can be written as products of functions of different variables. Moreover, the absorption coefficient contains multiple components. This is the parameter model proposed in $[30,33,57,66,94]$ to reconstruct chromophore concentrations from photoacoustic measurements.

We have the following uniqueness result with the multi-spectral data.

COROLlary 4.1. Let $\left(\Upsilon, \sigma_{a}, \sigma_{s}\right)$ and $\left(\tilde{\Upsilon}, \tilde{\sigma}_{a}, \tilde{\sigma}_{s}\right)$ be two sets of coefficients of the form (4.3), satisfying the regularity assumptions in (A1). Assume that we have data from $M(\geq K)$ different wavelengths $\left\{\lambda_{m}\right\}_{m=1}^{M}$ such that the matrix $\boldsymbol{\alpha}=\left(\alpha_{k}\left(\lambda_{m}\right)\right), 1 \leq$ $k \leq K, 1 \leq m \leq M$ has rank $K$. Let $\mathcal{A}_{\lambda}$ be the albedo operator that depends on wavelength. Then $\mathcal{A}_{\lambda}=\tilde{\mathcal{A}}_{\lambda}$ implies $\left\{\Upsilon(\mathbf{x}),\left\{\sigma_{a}^{k}(\mathbf{x})\right\}_{k=1}^{K}, \sigma_{s}(\mathbf{x})\right\}=\left\{\tilde{\Upsilon}(\mathbf{x}),\left\{\tilde{\sigma}_{a}^{k}(\mathbf{x})\right\}_{k=1}^{K}, \tilde{\sigma}_{s}(\mathbf{x})\right\}$.

Proof. Corollary 3.2 implies that we can uniquely reconstruct

$$
\mu(\mathbf{x}, \lambda)=\gamma(\lambda) \Upsilon(\mathbf{x}) \sigma_{a}(\mathbf{x}, \lambda), \quad \sigma(\mathbf{x}, \lambda)=\sigma_{a}(\mathbf{x}, \lambda)+\beta(\lambda) \sigma_{s}(\mathbf{x}) .
$$

Take two wavelengths $\lambda_{1}$ and $\lambda_{2}$. We then have

$$
\begin{gathered}
\frac{\mu\left(\mathbf{x}, \lambda_{1}\right)}{\mu\left(\mathbf{x}, \lambda_{2}\right)}=\frac{\gamma\left(\lambda_{1}\right) \sigma_{a}\left(\mathbf{x}, \lambda_{1}\right)}{\gamma\left(\lambda_{2}\right) \sigma_{a}\left(\mathbf{x}, \lambda_{2}\right)}, \\
\beta\left(\lambda_{2}\right) \sigma\left(\mathbf{x}, \lambda_{1}\right)-\beta\left(\lambda_{1}\right) \sigma\left(\mathbf{x}, \lambda_{2}\right)=\beta\left(\lambda_{2}\right) \sigma_{a}\left(\mathbf{x}, \lambda_{1}\right)-\beta\left(\lambda_{1}\right) \sigma_{a}\left(\mathbf{x}, \lambda_{2}\right) .
\end{gathered}
$$

If $\beta\left(\lambda_{1}\right) \gamma\left(\lambda_{1}\right) \mu\left(\mathbf{x}, \lambda_{2}\right)-\beta\left(\lambda_{2}\right) \gamma\left(\lambda_{2}\right) \mu\left(\mathbf{x}, \lambda_{1}\right) \neq 0$ at $\mathbf{x} \in \Omega$, we can solve the above system of equations to reconstruct $\sigma_{a}\left(\mathbf{x}, \lambda_{1}\right)$ and $\sigma_{a}\left(\mathbf{x}, \lambda_{2}\right)$. We then obtain $\Upsilon(\mathbf{x})$ and $\sigma_{s}(\mathbf{x})$. Once we know $\Upsilon(\mathbf{x})$ and $\sigma_{s}(\mathbf{x})$, we can uniquely reconstruct $\sigma_{a}(\mathbf{x}, \lambda)$ for any $\lambda \in \Lambda$. Now select $\left\{\lambda_{m}\right\}_{m=1}^{M}$ from $\Lambda$ such that the matrix $\boldsymbol{\alpha}=\left(\alpha_{k}\left(\lambda_{m}\right)\right), 1 \leq k \leq$ $K, 1 \leq m \leq M$ has rank $K$. We can reconstruct $\left\{\sigma_{a}^{k}(\mathbf{x})\right\}_{k=1}^{K}$ by solving the system $\sum_{k=1}^{K} \alpha_{k}\left(\lambda_{m}\right) \sigma_{a}^{k}(\mathbf{x})=\sigma\left(\mathbf{x}, \lambda_{m}\right), 1 \leq m \leq M$. This completes the proof.

4.2. Reconstruction methods. The linearized and nonlinear reconstruction methods proposed in the previous section can be adapted to use the multi-spectral interior data. We will not repeat the whole algorithm again but just highlight the main modifications here for the linearized reconstruction with Born approximation.

We can build an analogue of (3.11):

$$
\frac{H_{\lambda}}{\gamma(\lambda) \sigma_{a 0}\left\langle U_{\lambda}\right\rangle}(\mathbf{x})=\mathcal{I}(\Upsilon)+\mathcal{L}_{\lambda}^{a}\left(\Upsilon \tilde{\sigma}_{a}\right)+\mathcal{L}_{\lambda}^{s}\left(\Upsilon \tilde{\sigma}_{s}\right),
$$


where $\mathcal{I}$ is the identity operator and the operators $\mathcal{L}^{a}$ and $\mathcal{L}^{s}$ are defined, respectively, as

$$
\begin{aligned}
& \mathcal{L}_{\lambda}^{a}\left(\Upsilon \tilde{\sigma}_{a}\right)=\frac{\Upsilon \tilde{\sigma}_{a}}{\sigma_{a 0}}+\int_{\Omega} \Upsilon(\mathbf{y}) \tilde{\sigma}_{a}(\mathbf{y}, \lambda) \frac{\left\langle U_{\lambda} G_{\lambda}\right\rangle_{\mathbf{v}}}{\left\langle U_{\lambda}\right\rangle_{\mathbf{v}}}(\mathbf{y} ; \mathbf{x}) d \mathbf{y} \\
& \mathcal{L}_{\lambda}^{s}\left(\Upsilon \tilde{\sigma}_{s}\right)=-\alpha(\lambda) \int_{\Omega} \Upsilon(\mathbf{y}) \tilde{\sigma}_{s}(\mathbf{y}) \frac{\left\langle K\left(U_{\lambda}\right) G_{\lambda}\right\rangle_{\mathbf{v}}}{\left\langle U_{\lambda}\right\rangle_{\mathbf{v}}}(\mathbf{y} ; \mathbf{x}) d \mathbf{y} .
\end{aligned}
$$

Let us assume again that we collect the data for $N_{g}$ different illumination patterns and that for each illumination pattern we have data for $M$ different wavelengths $\left\{\lambda_{m}\right\}_{m=1}^{M}$. We denote by $H_{i, \lambda_{m}}, 1 \leq i \leq N_{g}, 1 \leq m \leq M$ the $i$ th data set at wavelength $\lambda_{m}$. The system of linear integral equations with these $N_{g} \times M$ data sets can be written as

$$
\left(\begin{array}{cccccc}
\mathcal{I} & \mathcal{L}_{1, \lambda_{1}}^{a} & 0 & \ldots & 0 & \mathcal{L}_{1, \lambda_{1}}^{s} \\
\vdots & \vdots & \vdots & & & \\
\mathcal{I} & 0 & 0 & \cdots & \mathcal{L}_{1, \lambda_{M}}^{a} & \mathcal{L}_{1, \lambda_{M}}^{s} \\
\vdots & \vdots & \vdots & & & \\
\mathcal{I} & \mathcal{L}_{i, \lambda_{1}}^{a} & 0 & \ldots & 0 & \mathcal{L}_{i, \lambda_{1}}^{s} \\
\vdots & \vdots & \vdots & & & \\
\mathcal{I} & 0 & 0 & \ldots & \mathcal{L}_{i, \lambda_{M}}^{a} & \mathcal{L}_{i, \lambda_{M}}^{s} \\
\vdots & \vdots & \vdots & & & \\
\mathcal{I} & \mathcal{L}_{N_{g}, \lambda_{1}}^{a} & 0 & \cdots & 0 & \mathcal{L}_{N_{g}, \lambda_{1}}^{s} \\
\vdots & \vdots & \vdots & & & \\
\mathcal{I} & 0 & 0 & \cdots & \mathcal{L}_{N_{g}, \lambda_{M}}^{a} & \mathcal{L}_{N_{g}, \lambda_{M}}^{s}
\end{array}\right)\left(\begin{array}{c}
\Upsilon \\
\tilde{\sigma}_{a}\left(\mathbf{x}, \lambda_{1}\right) \\
\vdots \\
\tilde{\sigma}_{a}\left(\mathbf{x}, \lambda_{m}\right) \\
\vdots \\
\tilde{\sigma}_{a}\left(\mathbf{x}, \lambda_{M}\right) \\
\tilde{\sigma}_{s}(\mathbf{x})
\end{array}\right)=\left(\begin{array}{c}
h_{1, \lambda_{1}} \\
\vdots \\
h_{1, \lambda_{M}} \\
\vdots \\
h_{i, \lambda_{1}} \\
\vdots \\
h_{i, \lambda_{M}} \\
\vdots \\
h_{N_{g}, \lambda_{1}} \\
\vdots \\
h_{N_{g}, \lambda_{M}}
\end{array}\right)
$$

with $\mathcal{L}_{i, \lambda_{m}}^{a}$ and $\mathcal{L}_{i, \lambda_{m}}^{s}$ being the evaluation of the operators defined in (4.8) and (4.9), respectively, at source $g_{i}$ and wavelength $\lambda_{m}$. If we introduce the notation

$$
\begin{aligned}
\mathcal{L}_{i}^{a} & =\operatorname{diag}\left(\mathcal{L}_{i, \lambda_{m}}^{a}, \ldots, \mathcal{L}_{i, \lambda_{m}}^{a}, \ldots, \mathcal{L}_{i, \lambda_{M}}^{a}\right), \\
\tilde{\sigma}_{a} & =\left(\tilde{\sigma}_{a}\left(\mathbf{x}, \lambda_{1}\right), \ldots, \tilde{\sigma}_{a}\left(\mathbf{x}, \lambda_{m}\right), \ldots, \tilde{\sigma}_{a}\left(\mathbf{x}, \lambda_{M}\right)\right)^{T}, \\
h_{i} & =\left(h_{i, \lambda_{1}}, \ldots, h_{i, \lambda_{m}}, \ldots, h_{i, \lambda_{M}}\right)^{T},
\end{aligned}
$$

then this system is exactly the same form as (3.14). We solve this linear system in the least-squares sense again in exactly the same ways as those presented in Section 3.2. Once $\left(\Upsilon(\mathbf{x}),\left\{\tilde{\sigma}_{a}\left(\mathbf{x}, \lambda_{m}\right)\right\}_{m=1}^{M}, \tilde{\sigma}_{s}(\mathbf{x})\right)$ are reconstructed, we reconstruct the coefficient components $\left\{\tilde{\sigma}_{a}^{k}\right\}_{k=1}^{K}$ by solving the following linear system (in least-squares sense) locally (i.e. at each spatial location $\mathbf{x} \in \Omega$ ):

$$
\left(\begin{array}{ccc}
\alpha_{1}\left(\lambda_{1}\right) & \cdots & \alpha_{K}\left(\lambda_{1}\right) \\
\vdots & \cdots & \vdots \\
\alpha_{1}\left(\lambda_{M}\right) & \cdots & \alpha_{K}\left(\lambda_{M}\right)
\end{array}\right)\left(\begin{array}{c}
\tilde{\sigma}_{a}^{1}(\mathbf{x}) \\
\vdots \\
\tilde{\sigma}_{a}^{K}(\mathbf{x})
\end{array}\right)=\left(\begin{array}{c}
\tilde{\sigma}_{a}\left(\mathbf{x}, \lambda_{1}\right) \\
\vdots \\
\tilde{\sigma}_{a}\left(\mathbf{x}, \lambda_{M}\right)
\end{array}\right) .
$$

\section{Numerical experiments}

We now present some numerical simulations with synthetic data to demonstrate the performance of the algorithms that we have presented in the previous sections. To simplify numerical computation, we consider only two-dimensional simulations, 
although the algorithms we have described are independent of spatial dimension. We will use both $\mathbf{x}$ and $(x, y)$ to denote a point on the plane. The computational domain is the square $\Omega=(0,2) \times(0,2)$. We denote by $\bar{\Omega} \equiv \Omega \cup \partial \Omega$. We also denote by $\left.\partial \Omega\right|_{L},\left.\partial \Omega\right|_{R},\left.\partial \Omega\right|_{B}$, and $\left.\partial \Omega\right|_{T}$ the left, right, bottom, and top sides of the boundary $\partial \Omega$, respectively. For instance, $\left.\partial \Omega\right|_{L}=\{(x, y) \mid x=0, y \in(0,2)\}$. The unit sphere $\mathbb{S}^{1}$ is parameterized by an angle $\theta \in[0,2 \pi)$ so that the direction vector $\mathbf{v}$ can be represented as $(\cos \theta, \sin \theta)$. As before, we will denote by $\langle u\rangle_{\mathbf{v}}$ and $\langle u\rangle_{\theta}$ the average of $u$ on $\mathbb{S}^{1}$.

To generate synthetic data, we solve the transport problem on a spatial mesh that is four times as fine as the mesh used to solve the inverse problem. We then compute $H$ in (1.3) by averaging in the four neighbor cells. This way, the interior data $H$ automatically contains noise due to the mismatch in spatial discretization. However, we will still call the data constructed in this way the "noiseless" data. In the case when the parameters that we are interested in are discontinuous in space, there is no way to exactly recover the discontinuity in the coefficients even with noiseless data.

To get noisy data, we add random noise in the following way. Let $\mathbf{H} \in \mathbb{R}^{N_{\Omega}}\left(N_{\Omega}\right.$ being the total number of grid points) be a vector of noiseless data on the grid, then the noisy data vector $\widetilde{\mathbf{H}} \in \mathbb{R}^{N_{\Omega}}$ is given by

$$
\widetilde{\mathbf{H}}=(\mathcal{I}+\epsilon \mathcal{N}) \mathbf{H}, \quad \mathcal{N}=\operatorname{diag}\left(X_{1}, \ldots, X_{N_{\Omega}}\right),
$$

where $X_{j}, j=1, \ldots, N_{\Omega}$ are independent identically distributed Gaussian random variables with zero mean and unit variance, and $\epsilon$ is the parameter that controls the noise level in the noisy data. For a particular value of the parameter $\epsilon$ we say that the noise level is $\epsilon \cdot 100 \%$. For example, for $\epsilon=0.05$ we say the noise level is $5 \%$.

To measure the error in the reconstruction of a quantity, say $\zeta$, we define the relative $l^{2}$ error $\mathcal{E}_{\zeta}$ of the reconstruction as

$$
\mathcal{E}_{\zeta}=\frac{\|\tilde{\zeta}-\zeta\|_{l^{2}}}{\|\zeta\|_{l^{2}}}
$$

where $\zeta \in \mathbb{R}^{N_{\Omega}}$ and $\tilde{\boldsymbol{\zeta}} \in \mathbb{R}^{N_{\Omega}}$ are the vectors containing the true and reconstructed quantity, respectively. For instance, $\mathcal{E}_{\sigma_{a}}$ is the relative $L^{2}$ error in the reconstruction of $\sigma_{a}$.

5.1. Reconstructions in non-scattering media. In this section we present some numerical simulations in non-scattering media following the reconstruction methods presented in Section 2. To do the reconstruction, we cover the domain with $100 \times 100$ cells of uniform size whose nodes are given as

$$
\Omega_{\Delta}=\left\{\mathbf{x}_{i, j}=\left(x_{i}, y_{j}\right) \mid x_{i}=i \Delta x, y_{j}=j \Delta y, i, j=0,1, \ldots, 100\right\},
$$

with $\Delta x=\Delta y=0.02$. This is four times as fine as the grids used in next section for reconstructions in scattering media.

5.1.1. Reconstructing $\sigma_{a}$. We show now some reconstructions of the absorption coefficient.

In the first numerical simulation in this group, we perform a reconstruction of a piecewise constant absorption function using a collimated source located on the left side of the boundary pointing inside the domain. The source is $g(\mathbf{x}, \mathbf{v})=\chi_{\left.\partial \Omega\right|_{L}} \delta\left(\mathbf{v}-\mathbf{v}^{\prime}\right)$ with $\mathbf{v}^{\prime}=(1,0)$. The absorption function consists of a background $\sigma_{a}=0.1 \mathrm{~cm}^{-1}$ and three disk inclusions $\Omega_{1}=\{\mathbf{x} \in \Omega|| \mathbf{x}-(0.6,0.6) \mid \leq 0.2\}, \Omega_{2}=\{\mathbf{x} \in \Omega|| \mathbf{x}-(1.4,0.6) \mid \leq$ $0.2\}$, and $\Omega_{3}=\{\mathbf{x} \in \Omega|| \mathbf{x}-(1.0,1.5) \mid \leq 0.3\}$ with values $\sigma_{a \mid \Omega_{1}}=0.3 \mathrm{~cm}^{-1}, \sigma_{a \mid \Omega_{2}}=$ 

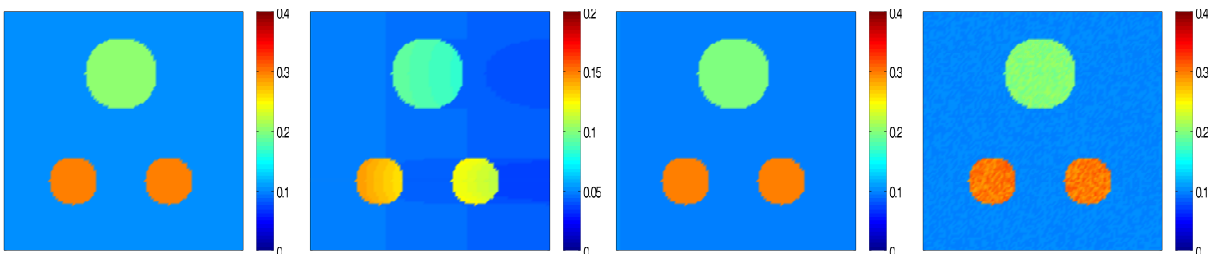

FIG. 5.1. Reconstructions of a piecewise constant absorption coefficient with a collimated source. Left to right: true absorption coefficient $\sigma_{a}$, interior data $H, \sigma_{a}$ reconstructed with noiseless data, and $\sigma_{a}$ reconstructed with noisy data (noise level $5 \%$ ).
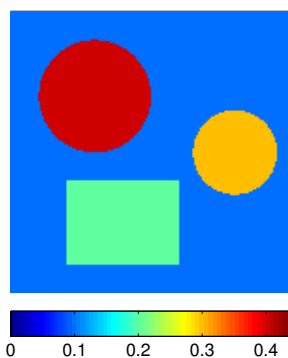
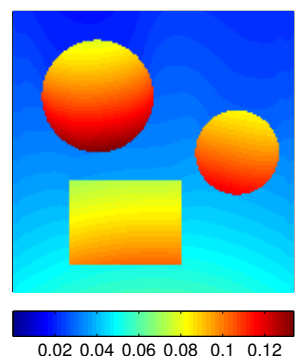
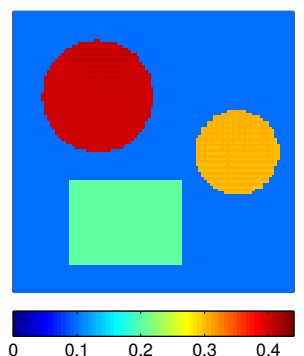
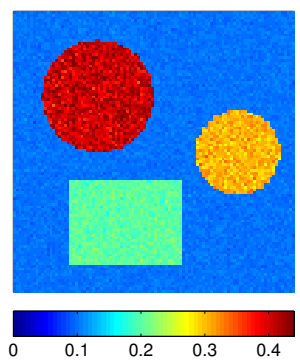

FIG. 5.2. Reconstructions of a piecewise constant absorption coefficient with cone-limited source. Left to right: true absorption coefficient $\sigma_{a}$, interior data $H, \sigma_{a}$ reconstructed with noiseless data and $\sigma_{a}$ reconstructed with noisy data (noise level $5 \%$ ).
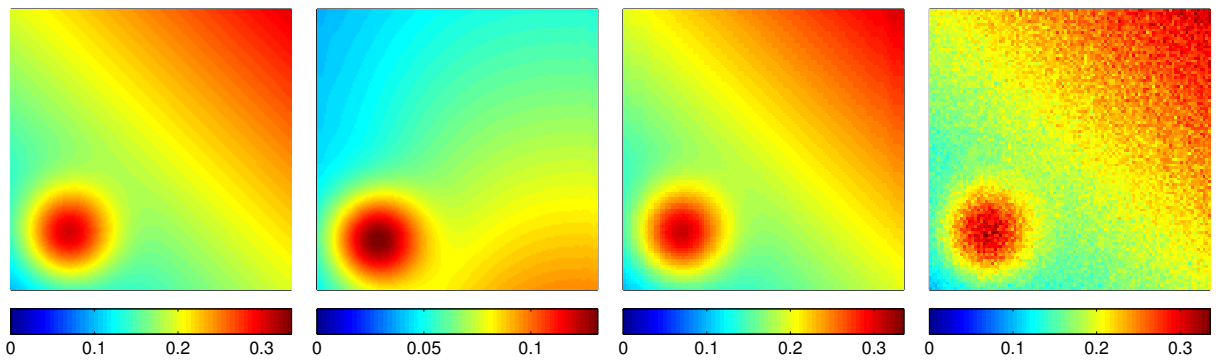

FIG. 5.3. Reconstructions of a smooth absorption coefficient with cone-limited source. Left to right: true absorption coefficient $\sigma_{a}$, interior data $H, \sigma_{a}$ reconstructed with noiseless data and $\sigma_{a}$ reconstructed with noisy data (noise level $5 \%$ ).

$0.3 \mathrm{~cm}^{-1}$, and $\sigma_{a \mid \Omega_{3}}=0.2 \mathrm{~cm}^{-1}$, respectively. The reconstruction results with noiseless and noisy data are presented in figure 5.1. The reconstruction is almost perfect when noiseless data is used. When noisy data $(\epsilon=0.05)$ is used, we can clearly see a degeneration of the quality of the reconstruction. However, the degeneration is very small, comparable to the noise level of the data. The relative $L^{2}$ error in the reconstructions are $\mathcal{E}_{\sigma_{a}}=0.3 \%$ and $\mathcal{E}_{\sigma_{a}}=2.8 \%$ in noiseless and noisy case, respectively.

Next we perform two reconstructions using a cone-limited source. The setup is as depicted in figure 2.2. The boundary condition $g(\mathbf{x}, \theta)$ corresponds to a uniform isotropic line source of unit intensity concentrated on a segment $\{(x, y) \mid x \in(0,2), y=$ $-2\}$, which results in $g(\mathbf{x}, \theta)$ being non-zero only on $\left.\partial \Omega\right|_{B}$. The half-aperture angle 
$\theta_{0}$ for such a source is $\pi / 4$, so we only keep track of the solution $u(x, y, \theta)$ for $\theta \in$ $[\pi / 4,3 \pi / 4]$. This segment is uniformly discretized with 50 nodes to generate the data and with 40 nodes to solve the inverse problem using the algorithm from Section 2.3.

We show in figure 5.2 the reconstruction of a piecewise-constant absorption coefficient with the cone-limited source. The absorption coefficient (in units of $\mathrm{cm}^{-1}$ ) is given by

$$
\sigma_{a}(\mathbf{x})=0.1+0.1 \chi_{\Omega_{1}}(\mathbf{x})+0.2 \chi_{\Omega_{2}}(\mathbf{x})+0.3 \chi_{\Omega_{3}}(\mathbf{x}),
$$

with the rectangular inclusion $\Omega_{1}=[0.4,1.2] \times[0.2,0.8]$, the smaller disk inclusion $\Omega_{2}=\{\mathbf{x} \in \Omega|| \mathbf{x}-(1.6,1.0) \mid \leq 0.3\}$, and the larger disk inclusion $\Omega_{3}=\{\mathbf{x} \in \Omega|| \mathbf{x}-$ $(0.6,1.4) \mid \leq 0.4\}$. Thus the absorption coefficient in the background is $\sigma_{a}=0.1 \mathrm{~cm}^{-1}$ while those in the three inclusions take the values $\left.\sigma_{a}\right|_{\Omega_{1}}=0.2 \mathrm{~cm}^{-1},\left.\sigma_{a}\right|_{\Omega_{2}}=0.3 \mathrm{~cm}^{-1}$, and $\left.\sigma_{a}\right|_{\Omega_{3}}=0.4 \mathrm{~cm}^{-1}$, respectively. The quality of the reconstruction is comparable to that in the previous numerical experiment in figure 5.1. The relative $L^{2}$ error in the reconstructions are $\mathcal{E}_{\sigma_{a}}=7.7 \%$ and $\mathcal{E}_{\sigma_{a}}=9.2 \%$ in the noiseless and noisy case, respectively.

Smoother absorption coefficients can be reconstructed with similar quality. To demonstrate this, we show in figure 5.3 the reconstruction of the absorption coefficient that is a sum of a Gaussian and linear functions given by

$$
\sigma_{a}(x, y)=A x+B y+C+D e^{\left((x-0.4)^{2}+(y-0.4)^{2}\right) / s_{D}^{2}},
$$

where the parameters $A, B, C, D$, and $s_{D}$ are chosen so that $0.1 \mathrm{~cm}^{-1} \leq \sigma_{a} \leq 0.3 \mathrm{~cm}^{-1}$. We performed reconstructions for many different choices of smooth absorption coefficients. The quality of the reconstruction in figure 5.3 is representative of the typical reconstruction quality that we obtained in those experiments.
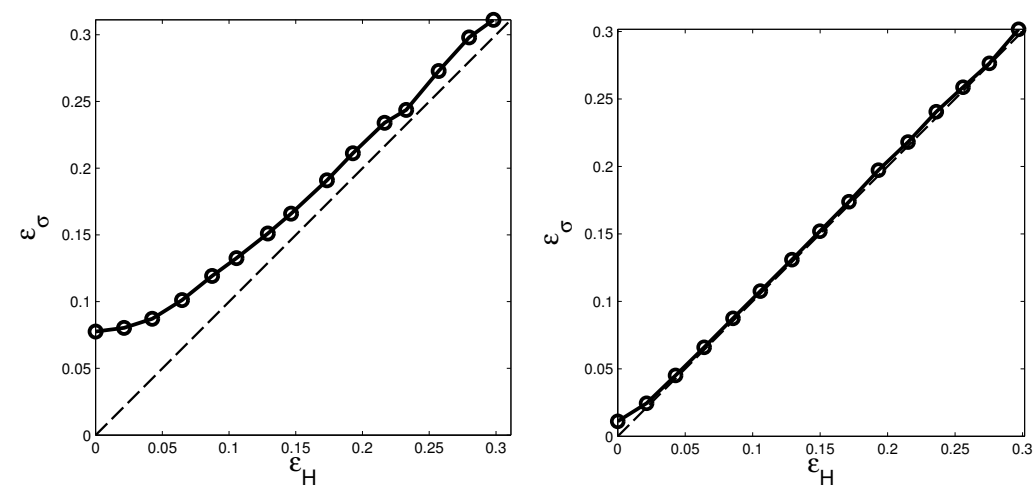

FIG. 5.4. Relative error $\mathcal{E}_{\sigma}$ in the reconstruction of the absorption coefficient with a cone-limited source versus noise level $\mathcal{E}_{H}$ in the data. Noise levels from 0 to $30 \%$. Left: reconstruction of piecewise constant absorption coefficient (5.3); Right: reconstruction of smooth absorption coefficient (5.4). Perfect linear stability $\mathcal{E}_{\sigma}=\mathcal{E}_{H}$ is shown as a dashed line for reference.

To characterize the stability of the reconstruction more precisely, we plot in figure 5.4 the relative $L^{2}$ error $\mathcal{E}_{\sigma}$ in the reconstruction of the absorption coefficients (5.3) and (5.4) versus the noise level $\mathcal{E}_{H}$ in the data used. Numerically the method appears to have linear stability, with the piecewise constant case being slightly worse than the smooth one. This is typically due to an imperfect resolution of the boundaries of the 
inclusions. Also, there is some residual error in the noiseless $(\epsilon=0)$ case due to the mismatch between the fine grid and the reconstruction grid.

5.1.2. Reconstructing $\left(\Upsilon, \sigma_{a}\right)$. To reconstruct both the Grüneisen coefficient and the absorption coefficient, we use data collected from two collimated sources located on the left and right sides of the boundary, respectively: $g_{1}(\mathbf{x}, \mathbf{v})=$ $\chi_{\left.\partial \Omega\right|_{L}} \delta\left(\mathbf{v}-\mathbf{v}^{\prime}\right)$ and $g_{2}(\mathbf{x}, \mathbf{v})=\chi_{\left.\partial \Omega\right|_{R}} \delta\left(\mathbf{v}+\mathbf{v}^{\prime}\right)$ with $\mathbf{v}^{\prime}=(1,0)$. The background ab-

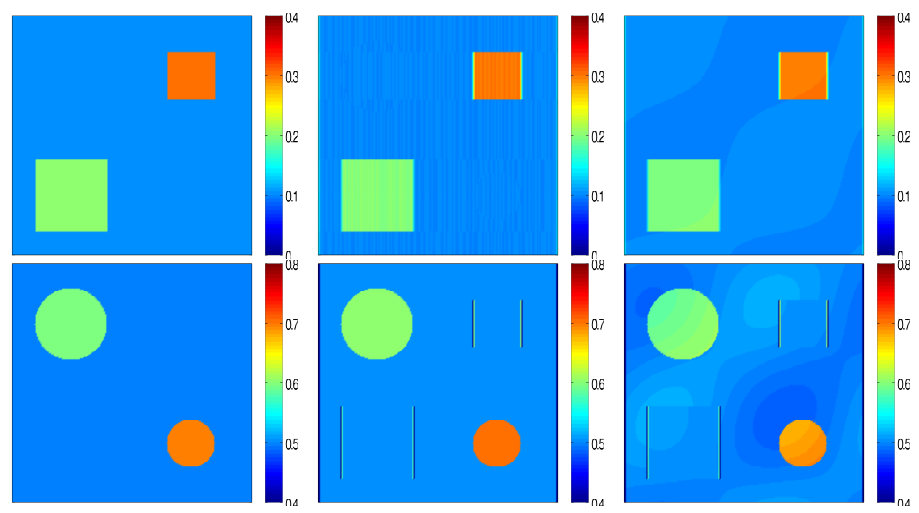

FIG. 5.5. Reconstructions of $\sigma_{a}$ (top row) and $\Upsilon$ (bottom row) using a pair of collimated sources. Left to right: true coefficients, coefficients reconstructed with noiseless data, and coefficients reconstructed with noisy data. The noisy data contains $5 \%$ random noise $(\epsilon=0.05)$.

sorption coefficient is $\sigma_{a}=0.1 \mathrm{~cm}^{-1}$ and the background Grüneisen coefficient is $\Upsilon=0.5$. There are four inclusions: two for the absorption coefficient located in $\Omega_{1}=\{\mathbf{x} \in \Omega|| \mathbf{x}-(0.5,0.5) \mid \leq 0.3\}$ and $\Omega_{2}=\{\mathbf{x} \in \Omega|| \mathbf{x}-(1.5,1.5) \mid \leq 0.2\}$, respectively, and two for the Grüneisen coefficient located in $\Omega_{3}=\{\mathbf{x} \in \Omega|| \mathbf{x}-(0.5,1.5) \mid \leq 0.2\}$ and $\Omega_{4}=\{\mathbf{x} \in \Omega|| \mathbf{x}-(1.5,0.5) \mid \leq 0.3\}$, respectively. The coefficients inside the inclusions are $\left.\sigma_{a}\right|_{\Omega_{1}}=0.2 \mathrm{~cm}^{-1},\left.\sigma_{a}\right|_{\Omega_{2}}=0.3 \mathrm{~cm}^{-1},\left.\Upsilon\right|_{\Omega_{3}}=0.6$, and $\left.\Upsilon\right|_{\Omega_{4}}=0.7$, respectively. We perform the reconstruction with both noiseless data and noisy data polluted with $5 \%$ additive random noise. The reconstruction results are presented in figure 5.5. Other than the phantom inclusions in the reconstructed Grüneisen coefficient at the locations of the inclusions of the absorption coefficient, the quality of the reconstructions is very high, and is comparable to the previous reconstructions in the cases of one unknown coefficient. The relative $L^{2}$ error in the reconstructions are $\left(\mathcal{E}_{\Upsilon}=2.8 \%, \mathcal{E}_{\sigma_{a}}=3.2 \%\right)$ and $\left(\mathcal{E}_{\Upsilon}=3.8 \%, \mathcal{E}_{\sigma_{a}}=4.4 \%\right)$ in the noiseless and noisy cases, respectively. The phantom inclusions in the reconstructed Grüneisen coefficients are caused by the inaccuracy of the reconstruction of the absorption coefficient at the boundary of the square inclusions. This inaccuracy originates from the differentiation of the quantity $\ln \frac{H_{2}}{H_{1}}$, which contains noise coming from mismatch between the forward and inversion grids. For the reconstruction from noisy data, the noise added to the synthetic data is random but has only low frequency components. If the data contain very high frequency components as in the previous cases, numerical differentiation of the quantity $\ln \frac{H_{2}}{H_{1}}$ in the algorithm would yield even larger noise in the reconstruction that would bury the true coefficients. Averaging from multiple reconstructions would be necessary to get a clean image. We would not address this issue in detail now but leave it to future study. 
5.2. Reconstructions in scattering media. Here we present some numerical simulations for scattering media, i.e. the case when $\sigma_{s} \neq 0$. In this case, we do not have analytical reconstruction formulas to work with. We thus use the linearized reconstruction and minimization-based nonlinear reconstruction schemes.

5.2.1. Numerical setup. The transport equations are solved with a finite volume scheme for the spatial variable and a discrete ordinate method for the angular variable. The domain is covered by $50 \times 50$ cells of uniform size whose nodes are given by

$$
\Omega_{h}=\left\{\mathbf{x}_{i, j}=\left(x_{i}, y_{j}\right) \mid x_{i}=i \Delta x, y_{j}=j \Delta y, i, j=0,1, \ldots, 50\right\},
$$

with $\Delta x=\Delta y=0.04$. We discretize $\mathbb{S}^{1}$ into 128 uniformly distributed directions with identical quadrature weight:

$$
\mathbb{S}_{\Delta \theta}^{1}=\left\{\mathbf{v}_{k}: \mathbf{v}_{k}=(k-1) * \Delta \theta, k=1, \ldots, 128\right\},
$$

where $\Delta \theta=2 \pi / 128$. Note that this discretization is not as fine as that used in the previous case when scattering is not present in the problem. This is only done here to save computational cost. It should not be regarded as a limitation of the reconstruction algorithms for scattering media.

The scattering kernel is chosen as the Henyey-Greenstein phase function $[9,54,97]$

$$
\mathcal{K}\left(\mathbf{v}, \mathbf{v}^{\prime}\right)=\frac{1}{2 \pi} \frac{1-\eta^{2}}{\left(1+\eta^{2}-2 \eta \mathbf{v} \cdot \mathbf{v}^{\prime}\right)^{3 / 2}},
$$

where $\eta \in[0,1]$ is the anisotropy factor, which measures how peaked forward the phase function is. The larger $\eta$ is, the more forward the scattering. The anisotropy factor is often used to define the so-called effective scattering coefficient through $\sigma_{s}^{\prime}=(1-\eta) \sigma_{s}$.

For more details on the forward solver, we refer to our previous publications $[78$, 79].

5.2.2. Reconstructing $\sigma_{a}$. We first reconstruct the absorption coefficient, assuming that both the scattering coefficient and the Grüneisen coefficients are known. The setup is as follows. The medium consists of a homogeneous background absorbing medium with absorption coefficient $\sigma_{a}=0.2 \mathrm{~cm}^{-1}$ and two absorbing inclusions. The first inclusion occupies $\Omega_{1}=[0.4,0.8] \times[0.4,0.8]$ with the absorption coefficient $\sigma_{a}=0.3 \mathrm{~cm}^{-1}$. The second inclusion occupies $\Omega_{2}=[1.2,1.6] \times[1.2,1.6]$ with the absorption coefficient $\sigma_{a}=0.1 \mathrm{~cm}^{-1}$. We first performed two reconstructions with the linearization method: a reconstruction in an isotropically scattering medium with $\eta=0, \sigma_{s}=8 \mathrm{~cm}^{-1}$ and a reconstruction in an anisotropically scattering medium with $\eta=0.9, \sigma_{s}=80 \mathrm{~cm}^{-1}$. The Grüneisen coefficient is always $\Upsilon=0.5$. The synthetic data used in these reconstructions is noiseless in the sense described at the beginning of this section. The results of the reconstructions are shown in figure 5.6. The quality of the reconstructions is very high (although shown on a coarser grid than those in the previous section). The relative $L^{2}$ error in the reconstructions are $\mathcal{E}_{\sigma_{a}}=2.8 \%$ (for Born reconstruction in isotropic medium), $\mathcal{E}_{\sigma_{a}}=3.0 \%$ (for Born reconstruction in anisotropic medium), $\mathcal{E}_{\sigma_{a}}=3.0 \%$ (for nonlinear reconstruction in isotropic medium), and $\mathcal{E}_{\sigma_{a}}=3.2 \%$ (for nonlinear reconstruction in isotropic medium), respectively. Note that the fast decay of field from the line source makes the second inclusion hardly visible in the data $H=\Upsilon \sigma_{a}\langle u\rangle_{\mathbf{v}}$ plots. This is one of the main reason that the quantitative step of PAT is needed. The reconstructions using the nonlinear reconstruction algorithm are presented in the right column of figure 5.6. 
5.2.3. Reconstructing $\left(\Upsilon, \sigma_{a}\right)$. We now perform numerical simulations where we reconstruct both the Grüneisen coefficient and the absorption coefficient. The scattering coefficient is fixed at $\sigma_{s}=8 \mathrm{~cm}^{-1}$ and the anisotropic factor $\eta=0$. The
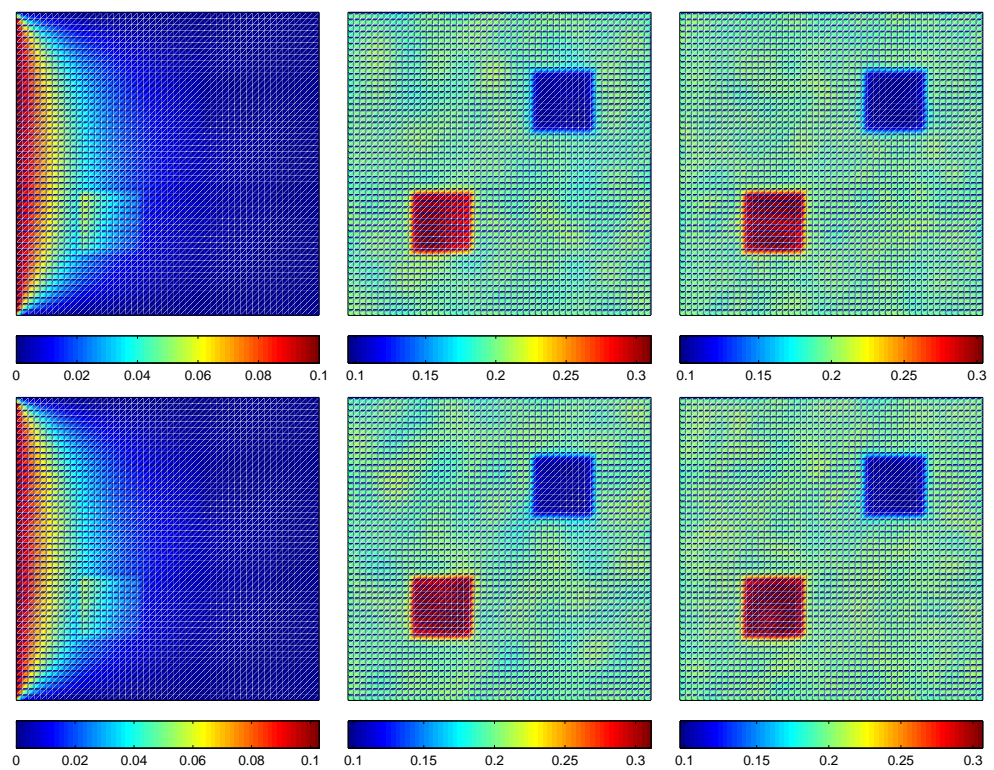

FIG. 5.6. Reconstructions of the absorption coefficient. Top row: the data $H=\Upsilon_{\sigma_{a}}\langle u\rangle_{\mathbf{v}}$ (left), $\sigma_{a}$ reconstructed using Born approximation (middle) and $\sigma_{a}$ reconstructed using nonlinear iteration (right) in an isotropic medium; Bottom row: same as the top row but in an anisotropic medium.
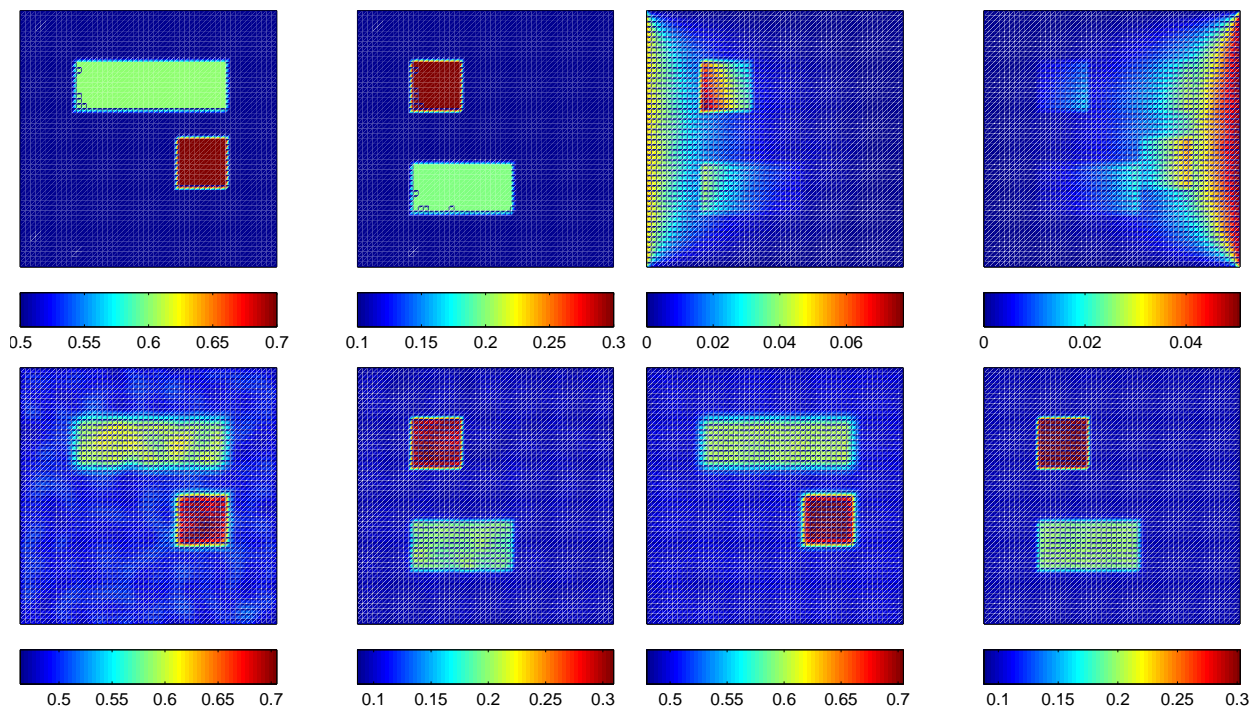

FIG. 5.7. Reconstructions of the Grüneisen and the absorption coefficients. Top left: true coefficients $\left(\Upsilon, \sigma_{a}\right)$ Top right: two data sets $\left(H_{1}, H_{2}\right)$ used in the reconstruction; Bottom left: reconstructed $\left(\Upsilon, \sigma_{a}\right)$ with two data sets; Bottom right: reconstructed $\left(\Upsilon, \sigma_{a}\right)$ with eight data sets. 
background absorption coefficient is $\sigma_{a}=0.1 \mathrm{~cm}^{-1}$ and the background Grüneisen coefficient is $\Upsilon=0.5$. There are four inclusions: two for the absorption coefficient located in $\Omega_{1}=[0.4,1.2] \times[0.4,0.8]$ and $\Omega_{2}=[0.4,0.8] \times[1.2,1.6]$, respectively, and two for the Grüneisen coefficient located in $\Omega_{3}=[0.4,1.6] \times[1.2,1.6]$ and $\Omega_{4}=[1.2,1.6] \times[0.6,1.0]$, respectively. The coefficients inside the inclusions are $\left.\sigma_{a}\right|_{\Omega_{1}}=0.2 \mathrm{~cm}^{-1},\left.\sigma_{a}\right|_{\Omega_{2}}=0.3$ $\mathrm{cm}^{-1},\left.\Upsilon\right|_{\Omega_{3}}=0.6$, and $\left.\Upsilon\right|_{\Omega_{4}}=0.7$, respectively. We perform the reconstruction with data polluted with $5 \%$ additive random noise added in the same way as in the previous section. The coefficients $\left(\Gamma, \sigma_{a}\right)$ reconstructed with two data sets generated with sources $g_{1}(\mathbf{x}, \mathbf{v})=\chi_{\partial \Omega_{L}}$ and $g_{2}(\mathbf{x}, \mathbf{v})=\chi_{\partial \Omega_{R}}$ are shown in figure 5.7 (the left two plots in the bottom row). The relative $L^{2}$ errors in the reconstructions are $\mathcal{E}_{\Upsilon}=5.3 \%$ and $\mathcal{E}_{\sigma_{a}}=4.5 \%$, respectively. The quality of the reconstruction is slightly lower than that in the reconstructions in the non-scattering regime, such as those shown in figure 5.5. It can be improved by averaging out noise in the data through the usage of additional data sets. This is demonstrated in figure 5.7 (the right two plots in the bottom row), where we show the reconstruction of the coefficients $\left(\Upsilon, \sigma_{a}\right)$ using eight data sets. The eight sources used are the rotation of the source $g_{1}(\mathbf{x})=\chi_{\partial \Omega_{L}}$ and $g_{2}=\chi_{\partial \Omega_{L}} \delta(\mathbf{v}-(1,0))$ over $0, \pi / 2, \pi$, and $3 \pi / 2$ around the center of the square domain $\Omega$. The relative $L^{2}$ errors in the reconstructions in this case are $\mathcal{E}_{\Upsilon}=3.3 \%$ and $\mathcal{E}_{\sigma_{a}}=3.5 \%$, respectively.
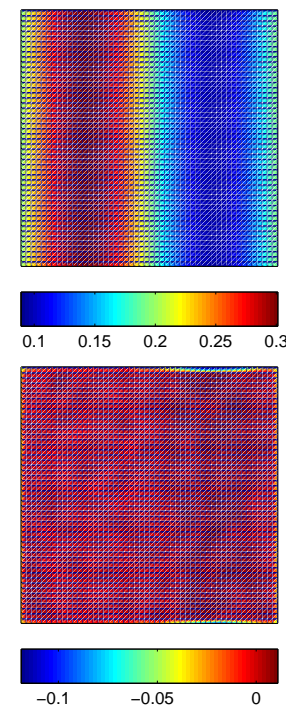
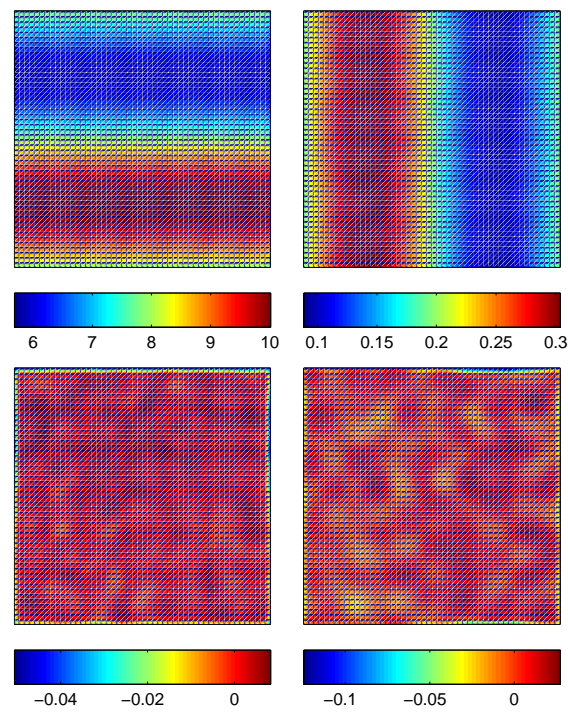
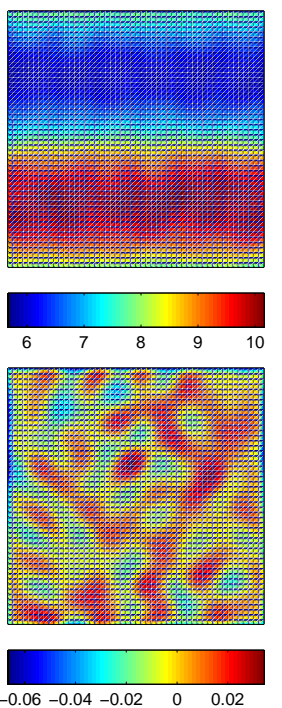

FIG. 5.8. Reconstructed absorption and scattering coefficients given in (5.6). Top row: ( $\sigma_{a}$, $\left.\sigma_{s}\right)$ reconstructed with noiseless (left two plots) and noisy (right two plots) data; Bottom row: the corresponding relative differences between reconstructed and real coefficients.

5.2.4. Reconstructing $\left(\sigma_{a}, \sigma_{s}\right)$. We now fix the Grüneisen coefficient $\Upsilon=1$ and attempt to reconstruct both the absorption and the scattering coefficient. We show in figure 5.8 the reconstructions of the objective absorption and scattering coefficients:

$$
\sigma_{a}(x, y)=0.2+0.1 \sin (\pi x), \quad \sigma_{s}(x, y)=8.0+2.0 \sin (\pi y) .
$$


Shown are the reconstructed $\left(\sigma_{a}, \sigma_{s}\right)$ (top row) with eight noiseless and noisy data sets and the corresponding pointwise relative error (bottom row), defined for $\sigma_{a}$ (resp. $\left.\sigma_{s}\right)$ as $\frac{\tilde{\sigma}_{a}-\sigma_{a}}{\sigma_{a}}$ (resp. $\frac{\tilde{\sigma}_{s}-\sigma_{s}}{\sigma_{s}}$ ) in the reconstructions. The maximal pointwise relative errors are $\left(\left\|\frac{\tilde{\sigma}_{a}-\sigma_{a}}{\sigma_{a}}\right\|_{L^{\infty}},\left\|\frac{\tilde{\sigma}_{s}-\sigma_{s}}{\sigma_{s}}\right\|_{L^{\infty}}\right)=(12.4 \%, 5.3 \%),\left(\left\|\frac{\tilde{\sigma}_{a}-\sigma_{a}}{\sigma_{a}}\right\|_{L^{\infty}},\left\|\frac{\tilde{\sigma}_{s}-\sigma_{s}}{\sigma_{s}}\right\|_{L^{\infty}}\right)=$ $(13.6 \%, 7.2 \%)$ in noise-free and noisy cases, respectively. Except for the relatively large error on some parts of the boundary, the error in the reconstructions is comparable to the noise level in the data. We observe that this is also confirmed in the reconstruction of piecewise constant coefficients such as those shown in figure 5.9. The piecewise constant absorption coefficient and the smooth scattering coefficient are given as

$$
\sigma_{a}(x, y)=0.2+0.1 \sin (\pi x), \quad \sigma_{s}(x, y)=8.0+2.0 \sin (\pi y),
$$

where the first absorbing inclusion is located in $\Omega_{1}=[0.4,0.8] \times[0.8,1.2]$ and the second absorbing inclusion is located in $\Omega_{2}=[1.4,1.6] \times[0.2,1.8]$. For piecewise constant coefficients, the reconstruction error occurs on the boundary of the inclusions. This is true also in the noiseless data case due to the fact that the synthetic data is generated by averaging quantities on a finer mesh. The Tikhonov regularization we employed in the numerical schemes also contribute to the smoothing on the boundary of the inclusions. The maximal pointwise relative errors are $\left(\left\|\frac{\tilde{\sigma}_{a}-\sigma_{a}}{\sigma_{a}}\right\|_{L^{\infty}},\left\|\frac{\tilde{\sigma}_{s}-\sigma_{s}}{\sigma_{s}}\right\|_{L^{\infty}}\right)=(19.6 \%, 5.6 \%)$ and $\left(\left\|\frac{\tilde{\sigma}_{a}-\sigma_{a}}{\sigma_{a}}\right\|_{L^{\infty}},\left\|\frac{\tilde{\sigma}_{s}-\sigma_{s}}{\sigma_{s}}\right\|_{L^{\infty}}\right)=(20.8 \%, 7.7 \%)$ in the noise-free and noisy cases, respectively.
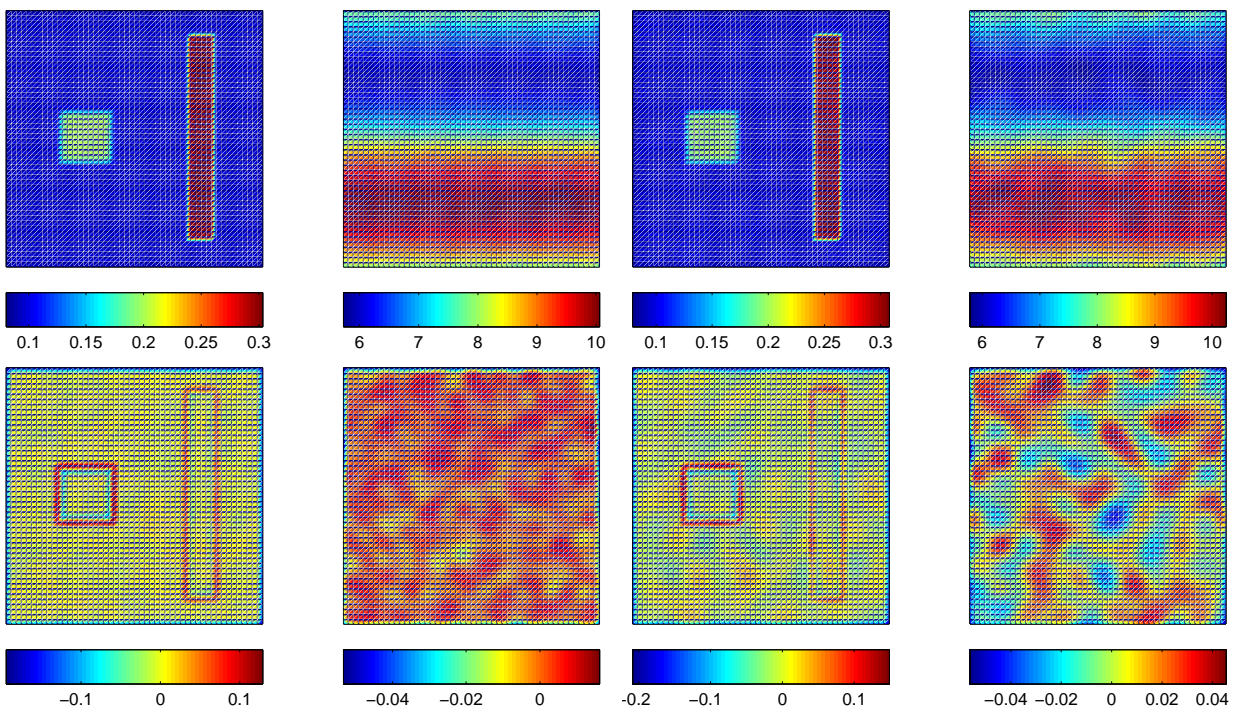

FIG. 5.9. Reconstructed absorption and scattering coefficients given in (5.7). Top row: ( $\sigma_{a}$, $\left.\sigma_{s}\right)$ reconstructed with noiseless (left two plots) and noisy (right two plots) data; Bottom row: the corresponding relative differences between reconstructed and real coefficients.

5.2.5. Reconstructing $\left(\Upsilon, \sigma_{a}, \sigma_{s}\right)$ with multi-spectral data.

The last numerical simulation is devoted to the simultaneous reconstructions of all three coefficients with multi-spectral interior data. The coefficients take the forms given in (4.3). To simplify the presentation, we consider only an absorption coefficient that 

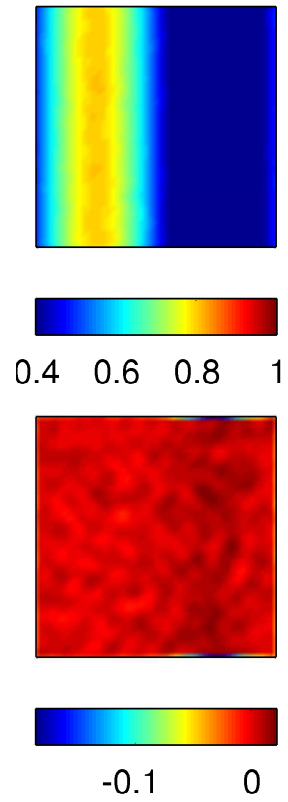
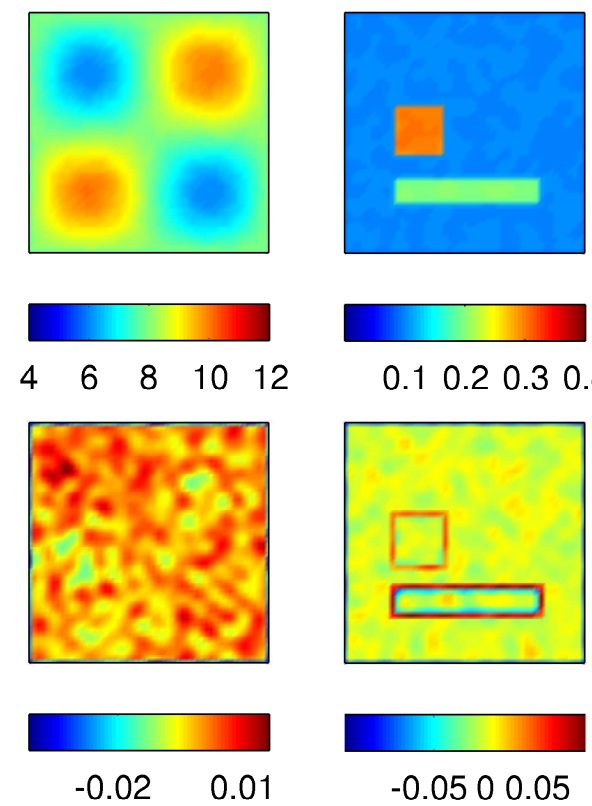

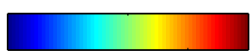

$\begin{array}{lllll}0.1 & 0.2 & 0.3 & 0.4\end{array}$
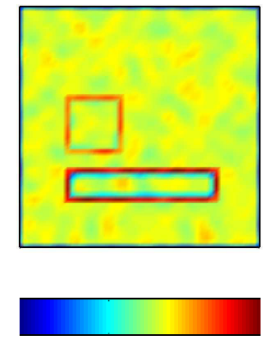

$-0.0500 .05$
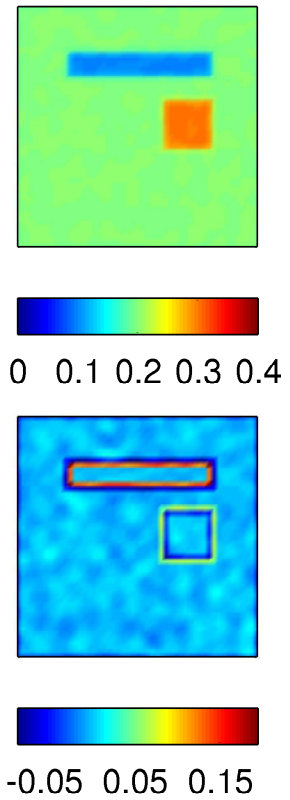

FIG. 5.10. Simultaneous reconstruction of the absorption and the scattering and the Grüneisen coefficients with multi-spectral data. Top row: reconstructed $\Upsilon, \sigma_{s}, \sigma_{a}^{1}$, and $\sigma_{a}^{2}$; Bottom row: the corresponding pointwise relative error in the reconstructions. The data used in this simulation are noiseless.
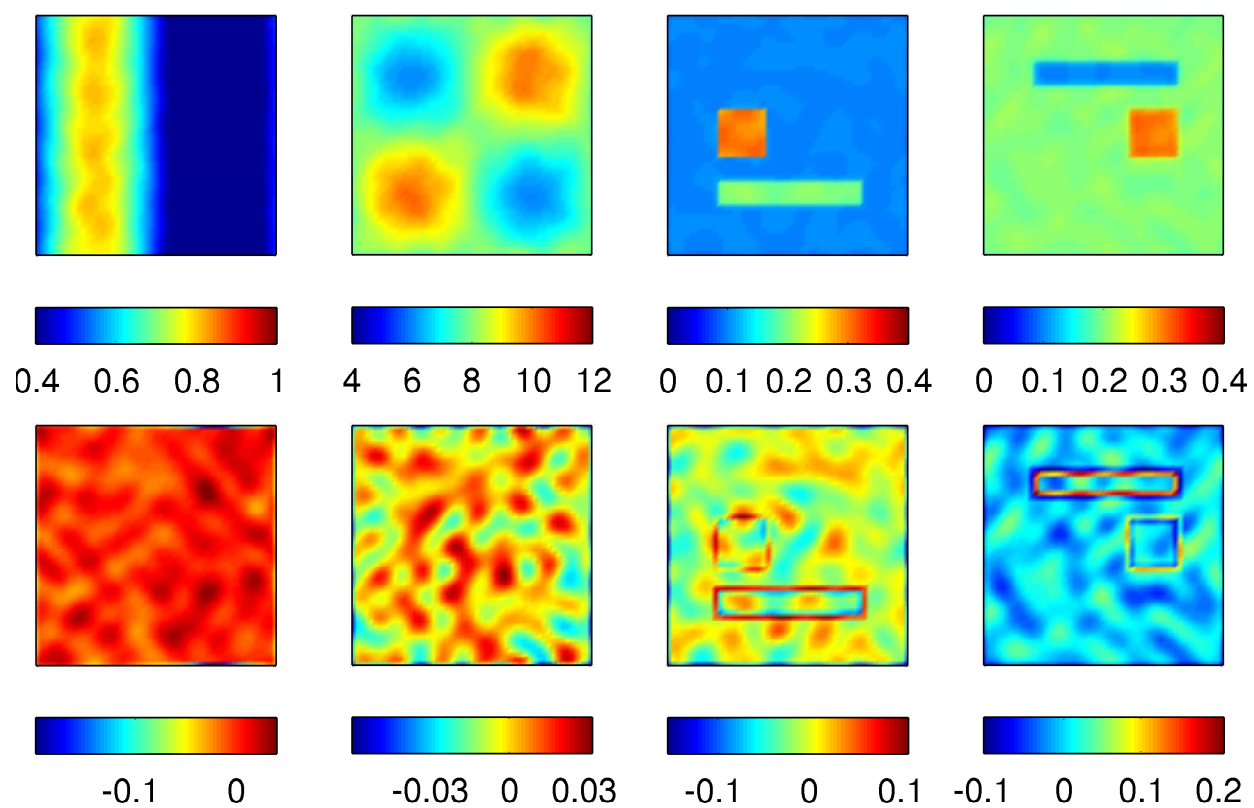

FIG. 5.11. Same as in figure 5.10 except that the data used contain $5 \%$ random noise. 
has two components, i.e. $K=2$. The spectral components of the coefficients are given as follows:

$$
\alpha_{1}(\lambda)=\frac{\lambda}{\lambda_{0}}, \quad \alpha_{2}(\lambda)=\frac{\lambda_{0}}{\lambda}, \quad \beta(\lambda)=\left(\frac{\lambda}{\lambda_{0}}\right)^{3 / 2}, \quad \gamma(\lambda)=1,
$$

where the normalization wavelength $\lambda_{0}$ is included to control the amplitude of the coefficients. These weight functions are by no means exactly what they should be in practical applications. However, the specific forms do not affect the results of the reconstruction. The spatial components of the coefficients are given as

$$
\begin{aligned}
& \Upsilon(\mathbf{x})=0.5+0.4 \tanh (4 x-4), \quad \sigma_{s}(\mathbf{x})=8.0+2.0 \sin (\pi x) \sin (\pi y), \\
& \sigma_{a}^{1}(\mathbf{x})=0.1+0.1 \chi_{\Omega_{1}}+0.2 \chi_{\Omega_{2}}, \quad \sigma_{a}^{2}(\mathbf{x})=0.2-0.1 \chi_{\Omega_{3}}+0.1 \chi_{\Omega_{4}},
\end{aligned}
$$

where $\Omega_{1}=[0.4,1.6] \times[0.4,0.6], \Omega_{2}=[0.4,0.8] \times[0.8,1.2], \Omega_{3}=[0.4,1.6] \times[1.4,1.6]$, and $\Omega_{4}=[1.2,1.6] \times[0.8,1.2]$. The anisotropic factor is $\eta=0$. We performed numerical reconstructions using four wavelength-dependent sources. For each source, we have data for four different wavelengths. The results of the reconstructions using noiseless data are presented in figure 5.10 and those using noisy data are shown in figure 5.11. We observe similar reconstruction qualities (as can be seen in the plots of the pointwise relative error) to the two-coefficient cases in the previous sections. The maximal pointwise relative errors $\left(\left\|\frac{\tilde{\Upsilon}-\Upsilon}{\Upsilon}\right\|_{L^{\infty}},\left\|\frac{\tilde{\sigma}_{s}-\sigma_{s}}{\sigma_{s}}\right\|_{L^{\infty}},\left\|\frac{\tilde{\sigma}_{a}^{1}-\sigma_{a}^{1}}{\sigma_{a}^{1}}\right\|_{L^{\infty}},\left\|\frac{\tilde{\sigma}_{a}^{2}-\sigma_{a}^{2}}{\sigma_{a}^{2}}\right\|_{L^{\infty}}\right)$ are $(19.7 \%, 3.4 \%, 7.2 \%, 18.4 \%),(22.9 \%, 4.4 \%, 11.9 \%, 21.2 \%)$ in the noise-free (figure 5.10$)$ and noisy (figure 5.11) cases, respectively.

\section{Concluding remarks}

We studied the quantitative photoacoustic tomography problem with the radiative transport model, aiming at reconstructing multiple physical coefficients simultaneously using the data collected from multiple illuminations. We showed that in nonscattering absorbing media, we can reconstruct both the absorption and the Grüneisen coefficients simultaneously in a stable manner using only two sets of interior data. Moreover, in this case we derived explicit reconstruction formulas for the problem with particular choices of illuminations (collimated, point, and cone-limited sources). In scattering media, we show, based on the result in [13], that one can stably reconstruct two of the absorption, the scattering, and the Grüneisen coefficients when more data, i.e., data given by the full albedo operator, is available. To reconstruct all three coefficients simultaneously, we proposed to use interior data collected at different optical wavelengths. We show that with some realistic a priori knowledge, mainly the knowledge of the spectral dependence of the coefficients, we can stably reconstruct the spatial component of all three coefficients simultaneously.

Besides the analytical reconstruction strategy for the non-scattering problem, we proposed a linearized reconstruction method based on Born approximation to the original inverse problem as well as a nonlinear reconstruction method based on numerical minimization techniques for QPAT for scattering media. We show numerically that the reconstruction is very stable. In fact, our numerical experiments showed that, assuming that the data measured with ultrasound is accurate enough, the nonlinear least-square formulation of the inverse transport problems with interior data problem behaves like a convex optimization problem and thus can be solved efficiently and accurately; see the numerical reconstructions in Section 5 .

There are many important issues in quantitative PAT that need to be addressed in the future. For instance, in the theory developed in $[14,18,16]$ for the diffusion model, one can reconstruct both the absorption and the scattering coefficients 
with only two "well-chosen" illuminations, with little a priori assumptions on the coefficients. It would be interesting to see how to generalize this to the radiative transport model (1.1) with $\sigma_{s} \neq 0$. An equally important question is whether or not we can derive any analytical reconstruction procedure, similar to those developed in diffusion-based theory $[18,14,16]$, for the inverse transport problem in scattering media.

On the numerical side, when the noise present in the data is significant, we need to regularize the reconstruction. The regularization we adopt here is the usual Tikhonov regularization which yields smooth solutions among all possibilities. In certain applications, we might know a priori that the coefficients to be reconstructed are piecewise constant, such as those presented in some of the numerical simulations in Section 5. In this case, alternative regularization strategies such as the total variation (TV) regularization might be more appropriate. In [14, 47, 48], $L^{1}$ regularization for non-smooth coefficients has been considered in the diffusion case. It would be interesting to see the performance of that in the transport case. We plan to explore this issue in the future.

Acknowledgment. We would like to thank the anonymous referees for their comments and suggestions on the first version of this paper. This work is partially supported by the National Science Foundation (NSF) through grant DMS-0914825.

\section{REFERENCES}

[1] M. Agranovsky, P. Kuchment, and L. Kunyansky, On reconstruction formulas and algorithms for the TAT and PAT tomography, in Photoacoustic Imaging and Spectroscopy, L.V. Wang (ed.), CRC Press, 89-101, 2009.

[2] M. Agranovsky and E.T. Quinto, Injectivity sets for the Radon transform over circles and complete systems of radial functions, J. Funct. Anal., 139, 383-414, 1996.

[3] G. Ambartsoumian, R. Gouia-Zarrad, and M. Lewis, Inversion of the circular Radon transform on an annulus, Inverse Prob., 26, 105015, 2010.

[4] H. Ammari, An Introduction to Mathematics of Emerging Biomedical Imaging, Springer, 2008.

[5] H. Ammari, E. Bossy, V. Jugnon, and H. Kang, Mathematical modelling in photo-acoustic imaging of small absorbers, SIAM Rev., 52, 677-695, 2010.

[6] H. Ammari, E. Bossy, V. Jugnon, and H. Kang, Reconstruction of the optical absorption coefficient of a small absorber from the absorbed energy density, SIAM J. Appl. Math., 71, 676-693, 2011.

[7] H. Ammari, E. Bretin, J. Garnier, and A. Wahab, Time reversal in attenuating acoustic media, Contemp. Math., 548, 151-163, 2011.

[8] H. Ammari, E. Bretin, V. Jugnon, and A. Wahab, Photo-acoustic imaging for attenuating acoustic media, Lecture Notes in Mathematics, 2035, 53-80, 2011.

[9] S.R. Arridge, Optical tomography in medical imaging, Inverse Probl., 15, R41-R93, 1999.

[10] S.R. Arridge and J.C. Schotland, Optical tomography: Forward and inverse problems, Inverse Prob., 123010, 25, 2009.

[11] G. Bal, Inverse transport theory and applications, Inverse Prob., 053001, 25, 2009.

[12] G. Bal, Hybrid inverse problems and internal information, in Inside Out: Inverse Problems and Applications, G. Uhlmann (ed.), Mathematical Sciences Research Institute Publications, Cambridge University Press, 2012.

[13] G. Bal, A. Jollivet, and V. Jugnon, Inverse transport theory of photoacoustics, Inverse Prob., 025011, 26, 2010.

[14] G. Bal and K. Ren, Multi-source quantitative PAT in diffusive regime, Inverse Prob., 075003, 27, 2011.

[15] G. Bal and K. Ren, Non-uniqueness results for a hybrid inverse problem, in Tomography and Inverse Transport Theory, G. Bal, D. Finch, P. Kuchment, J. Schotland, P. Stefanov, and G. Uhlmann (eds.), Contemp. Math., Amer. Math. Soc., Providence, RI, 559, 29-38, 2011. 
[16] G. Bal and K. Ren, On multi-spectral quantitative photoacoustic tomography in diffusive regime, Inverse Prob., 025010, 28, 2012.

[17] G. Bal and J.C. Schotland, Inverse scattering and acousto-optic imaging, Phys. Rev. Lett., 043902, 104, 2010.

[18] G. Bal and G. Uhlmann, Inverse diffusion theory of photoacoustics, Inverse Prob., 085010, $26,2010$.

[19] G. Bal and G. Uhlmann, Reconstructions of coefficients in scalar second-order elliptic equations from knowledge of their solutions, Commun. Pure Appl. Math., 2012.

[20] B. Banerjee, S. Bagchi, R.M. Vasu, and D. Roy, Quatitative photoacoustic tomography from boundary pressure measurements: Noniterative recovery of optical absorption coefficient from the reconstructed absorbed energy map, J. Opt. Soc. Am. A, 25, 2347-2356, 2008.

[21] A.Q. Bauer, R.E. Nothdurft, T.N. Erpelding, L.V. Wang, and J.P. Culver, Quantitative photoacoustic imaging: Correcting for heterogeneous light fluence distributions using diffuse optical tomography, J. Biomed. Optics, 096016, 16, 2011.

[22] P. Beard, Biomedical photoacoustic imaging, Interface Focus, 1, 602-631, 2011.

[23] A. Buehler, A. Rosenthal, T. Jetzfellner, A. Dima, D. Razansky, and V. Ntziachristos, Modelbased optoacoustic inversions with incomplete projection data, Med. Phys., 38, 1694, 2011.

[24] P. Burgholzer, G.J. Matt, M. Haltmeier, and G. Paltauf, Exact and approximative imaging methods for photoacoustic tomography using an arbitrary detection surface, Phys. Rev. E, 046706, 75, 2007.

[25] A.J. Chaudhari, F. Darvas, J.R. Bading, R.A. Moats, P.S. Conti, D.J. Smith, S.R. Cherry, and R.M. Leahy, Hyperspectral and multispectral bioluminescence optical tomography for small animal imaging, Phys. Med. Biol., 50, 5421-5441, 2005.

[26] A.X. Cong and G. Wang, Multispectral bioluminescence tomography: Methodology and simulation, Int. J. Biomed. Imag., 57614, 2006.

[27] T. Correia, A. Gibson, and J. Hebden, Identification of the optimal wavelengths for optical topography: A photon measurement density function analysis, J. Biomed. Optics, 056002, $15,2010$.

[28] B. Cox, J.G. Laufer, S.R. Arridge, and C. Beard, Quantitative spectroscopic photoacoustic imaging: A review, J. Biomed. Optics, 061202, 17, 2012.

[29] B.T. Cox, S.R. Arridge, and P.C. Beard, Photoacoustic tomography with a limited-aperture planar sensor and a reverberant cavity, Inverse Prob., 23, S95-S112, 2007.

[30] B.T. Cox, S.R. Arridge, and P.C. Beard, Estimating chromophore distributions from multiwavelength photoacoustic images, J. Opt. Soc. Am. A, 26, 443-455, 2009.

[31] B.T. Cox, S.R. Arridge, K. P. Köstli, and P.C. Beard, Two-dimensional quantitative photoacoustic image reconstruction of absorption distributions in scattering media by use of a simple iterative method, Appl. Optics, 45, 1866-1875, 2006.

[32] B.T. Cox and P.C. Beard, Photoacoustic tomography with a single detector in a reverberant cavity, J. Opt. Soc. Am. A, 125, 1426-1436, 2009.

[33] B.T. Cox, J.G. Laufer, and P.C. Beard, The challenges for quantitative photoacoustic imaging, Proc. of SPIE, 717713, 7177, 2009.

[34] B.T. Cox, T. Tarvainen, and S.R. Arridge, Multiple illumination quantitative photoacoustic tomography using transport and diffusion models, in Tomography and Inverse Transport Theory, G. Bal, D. Finch, P. Kuchment, J. Schotland, P. Stefanov, and G. Uhlmann (eds.), Contemporary Mathematics, Amer. Math. Soc., Providence, RI, 559, 1-12, 2011.

[35] R. Dautray and J.L. Lions, Mathematical Analysis and Numerical Methods for Science and Technology, Vol VI, Springer-Verlag, Berlin, 1993.

[36] X.L. Deán-Ben, D. Razansky, and V. Ntziachristos, The effects of acoustic attenuation in optoacoustic signals, Phys. Med. Biol., 56, 6129-6148, 2011.

[37] O. Dorn, A transport-backtransport method for optical tomography, Inverse Prob., 14, 11071130, 1998.

[38] O. Dorn, Scattering and absorption transport sensitivity functions for optical tomography, Optics Express, 7, 492-506, 2000.

[39] P. Elbau, O. Scherzer, and R. Schulze, Reconstruction formulas for photoacoustic sectional imaging, Inverse Prob., 28(4), 045004, 2012.

[40] D. Finch, M. Haltmeier, and Rakesh, Inversion of spherical means and the wave equation in even dimensions, SIAM J. Appl. Math., 68, 392-412, 2007.

[41] D. Finch and Rakesh, The spherical mean operator with centers on a sphere, Inverse Prob., 35, S37-S50, 2007.

[42] S.K. Finch, D. Patch, and Rakesh, Determining a function from its mean values over a family of spheres, SIAM J. Math. Anal., 35, 1213-1240, 2004.

[43] K.O. Friedrichs, Advanced Ordinary Differential Equations, Taylor \& Francis, New York, 
1985.

[44] H. Gao, S. Osher, and H. Zhao, Quantitative photoacoustic tomography, in Mathematical Modeling in Biomedical Imaging II: Optical, Ultrasound, and Opto-Acoustic Tomographies, H. Ammari (ed.), Lecture Notes in Mathematics, Springer, 2012.

[45] H. Gao and H. Zhao, Multilevel bioluminescence tomography based on radiative transfer equation. Part 1: l1 regularization, Optics Express, 18, 1854-1871, 2010.

[46] H. Gao and H. Zhao, Multilevel bioluminescence tomography based on radiative transfer equation. Part 2: Total variation and l1 data fidelity, Optics Express, 18, 2894-2912, 2010.

[47] H. Gao and H. Zhao, Simultaneous reconstruction of absorption and scattering with multiple optical excitations in quantitative photoacoustic tomography via Bregman method, J. Biomed. Opt., 1, 1, 2010.

[48] H. Gao, H. Zhao, and S. Osher, Bregman methods in quantitative photoacoustic tomography, CAM Report, UCLA, 10-42, 2010.

[49] T. Görner, R. Hielscher, and S. Kunis, Efficient and accurate computation of spherical mean values at scattered center points, Inverse Prob. Imag., 6(4), 645-661, 2012.

[50] M. Haltmeier, Inversion formulas for a cylindrical Radon transform, SIAM J. Imag. Sci., 4, 789-806, 2011.

[51] M. Haltmeier, A mollification approach for inverting the spherical mean Radon transform, SIAM J. Appl. Math., 71, 1637-1652, 2011.

[52] M. Haltmeier, O. Scherzer, P. Burgholzer, and G. Paltauf, Thermoacoustic computed tomography with large planer receivers, Inverse Prob., 20, 1663-1673, 2004.

[53] M. Haltmeier, T. Schuster, and O. Scherzer, Filtered backprojection for thermoacoustic computed tomography in spherical geometry, Math. Meth. Appl. Sci., 28, 1919-1937, 2005.

[54] L.G. Henyey and J.L. Greenstein, Diffuse radiation in the galaxy, Astrophys. J., 90, 70-83, 1941.

[55] Y. Hristova, Time reversal in thermoacoustic tomography - an error estimate, Inverse Prob., 055008, 25, 2009.

[56] Y. Hristova, P. Kuchment, and L. Nguyen, Reconstruction and time reversal in thermoacoustic tomography in acoustically homogeneous and inhomogeneous media, Inverse Prob., 055006, 24, 2008

[57] H.K. Kim, M. Flexman, D.J. Yamashiro, J.J. Kandel, and A.H. Hielscher, PDE-constrained multispectral imaging of tissue chromophores with the equation of radiative transfer, Biomed. Optics Express, 1, 812-824, 2010.

[58] R. Kowar and O. Scherzer, Photoacoustic imaging taking into account attenuation, Math. Model. Bio. Imag. II Lecture Notes in Mathematics, 2035/2012, 85-130, 2012.

[59] P. Kuchment, Mathematics of hybrid imaging. A brief review, in The Mathematical Legacy of Leon Ehrenpreis, I. Sabadini and D. Struppa (eds.), Springer Proceedings in Mathematics, 16, 183-208, 2012.

[60] P. Kuchment and L. Kunyansky, Mathematics of thermoacoustic tomography, Euro. J. Appl. Math., 19, 191-224, 2008.

[61] P. Kuchment and L. Kunyansky, Mathematics of thermoacoustic and photoacoustic tomography, in Handbook of Mathematical Methods in Imaging, O. Scherzer (ed.), SpringerVerlag, 817-866, 2010.

[62] L. Kunyansky, Explicit inversion formulae for the spherical mean Radon transform, Inverse Prob., 23, 373-383, 2007.

[63] L. Kunyansky, A series solution and a fast algorithm for the inversion of the spherical mean Radon transform, Inverse Prob., 23, S11-S20, 2007.

[64] L. Kunyansky, Thermoacoustic tomography with detectors on an open curve: An efficient reconstruction algorithm, Inverse Prob., 055021, 24, 2008.

[65] L. Kunyansky, Reconstruction of a function from its spherical (circular) means with the centers lying on the surface of certain polygons and polyhedra, Inverse Prob., 025012, 27, 2011.

[66] J. Laufer, B.T. Cox, E. Zhang, and P. Beard, Quantitative determination of chromophore concentrations from $2 d$ photoacoustic images using a nonlinear model-based inversion scheme, Applied Optics, 49, 1219-1233, 2010.

[67] C. Li and L. Wang, Photoacoustic tomography and sensing in biomedicine, Phys. Med. Biol., 54, R59-R97, 2009.

[68] F. Natterer, Photo-acoustic inversion in convex domains, Inverse Prob. Imaging, 6, 315-320, 2012.

[69] L.V. Nguyen, A family of inversion formulas in thermoacoustic tomography, Inverse Prob. Imaging, 3, 649-675, 2009.

[70] G. Paltauf, R. Nuster, and P. Burgholzer, Weight factors for limited angle photoacoustic 
tomography, Phys. Med. Biol., 54, 3303-3314, 2009.

[71] G. Paltauf, R. Nuster, M. Haltmeier, and P. Burgholzer, Experimental evaluation of reconstruction algorithms for limited view photoacoustic tomography with line detectors, Inverse Prob., 23, S81-S94, 2007.

[72] S.K. Patch and O. Scherzer, Photo- and thermo- acoustic imaging, Inverse Prob., 23, S1-S10, 2007.

[73] J. Qian, P. Stefanov, G. Uhlmann, and H. Zhao, An efficient Neumann-series based algorithm for thermoacoustic and photoacoustic tomography with variable sound speed, SIAM J. Imag. Sci., 4, 850-883, 2011.

[74] D. Razansky, M. Distel, C. Vinegoni, R. Ma, N. Perrimon, R.W. Köster, and V. Ntziachristos, Multispectral opto-acoustic tomography of deep-seated fluorescent proteins in vivo, Nature Photonics, 3, 412-417, 2009.

[75] D. Razansky and V. Ntziachristos, Hybrid photoacoustic fluorescence molecular tomography using finite-element-based inversion, Med. Phys., 34, 4293-4301, 2007.

[76] D. Razansky, C. Vinegoni, and V. Ntziachristos, Multispectral photoacoustic imaging of fluorochromes in small animals, Optics Lett., 32, 2891-2893, 2007.

[77] K. Ren, Recent developments in numerical techniques for transport-based medical imaging methods, Commun. Comput. Phys., 8, 1-50, 2010.

[78] K. Ren, G.S. Abdoulaev, G. Bal, and A.H. Hielscher, Algorithm for solving the equation of radiative transfer in the frequency domain, Optics Lett., 29, 578-580, 2004.

[79] K. Ren, G. Bal, and A.H. Hielscher, Frequency domain optical tomography based on the equation of radiative transfer, SIAM J. Sci. Comput., 28, 1463-1489, 2006.

[80] K. Ren, H. Gao, and H. Zhao, A hybrid reconstruction method for quantitative photoacoustic imaging, SIAM J. Imag. Sci., 2012.

[81] J. Ripoll and V. Ntziachristos, Quantitative point source photoacoustic inversion formulas for scattering and absorbing media, Phys. Rev. E, 031912, 71, 2005.

[82] A. Rosenthal, D. Razansky, and V. Ntziachristos, Fast semi-analytical model-based acoustic inversion for quantitative optoacoustic tomography, IEEE Trans. Med. Imag., 29, 1275$1285,2010$.

[83] P. Shao, B. Cox, and R.J. Zemp, Estimating optical absorption, scattering, and Grueneisen distributions with multiple-illumination photoacoustic tomography, Appl. Opt., 50, 31453154, 2011.

[84] S. Srinivasan, B.W. Pogue, S. Jiang, H. Dehghani, and K.D. Paulsen, Spectrally constrained chromophore and scattering near-infrared tomography provides quantitative and robust reconstruction, Applied Optics, 44, 1858-1869, 2005.

[85] P. Stefanov and G. Uhlmann, Thermoacoustic tomography with variable sound speed, Inverse Prob., 075011, 25, 2009.

[86] P. Stefanov and G. Uhlmann, Thermoacoustic tomography arising in brain imaging, Inverse Prob., 045004, 27, 2011

[87] P. Stefanov and G. Uhlmann, Recovery of a source term or a speed with one measurement and applications, Trans. Amer. Math. Soc., 365, 5737-5758, 2013.

[88] D. Steinhauer, A reconstruction procedure for thermoacoustic tomography in the case of limited boundary data, arXiv:0905.2954, 2009.

[89] D. Steinhauer, A uniqueness theorem for thermoacoustic tomography in the case of limited boundary data, arXiv:0902.2838v2, 2009.

[90] C. Tao and X. Liu, Reconstruction of high quality photoacoustic tomography with a limitedview scanning, Optics Express, 18, 2760-2766, 2010.

[91] B.E. Treeby, J.G. Laufer, E.Z. Zhang, F.C. Norris, M.F. Lythgoe, P.C. Beard, and B.T. Cox, Acoustic attenuation compensation in photoacoustic tomography: Application to high-resolution $3 d$ imaging of vascular networks in mice, in Photons Plus Ultrasound: Imaging and Sensing, A.A. Oraevsky and L.V. Wang (eds.), Y178992CY978992, 2011.

[92] B.E. Treeby, E.Z. Zhang, and B.T. Cox, Photoacoustic tomography in absorbing acoustic media using time reversal, Inverse Prob., 115003, 26, 2010.

[93] C.R. Vogel, Computational Methods for Inverse Problems, Frontiers in Applied Mathematics, SIAM, Philadelphia, 2002.

[94] J. Wang, S.C. Davis, S. Srinivasan, S. Jiang, B.W. Pogue, and K.D. Paulsen, Spectral tomography with diffuse near-infrared light: Inclusion of broadband frequency domain spectral data, J. Biomed. Optics, 041305, 13, 2008.

[95] L.V. Wang, Ultrasound-mediated biophotonic imaging: A review of acousto-optical tomography and photo-acoustic tomography, Disease Markers, 19, 123-138, 2004.

[96] L.V. Wang, Tutorial on photoacoustic microscopy and computed tomography, IEEE J. Sel. Topics Quantum Electron., 14, 171-179, 2008. 
[97] A.J. Welch and M.J.C. Van-Gemert, Optical-thermal Response of Laser Irradiated Tissue, Plenum Press, New York, 1995.

[98] D. Wu, C. Tao, and X. Liu, Photoacoustic tomography in scattering biological tissue by using virtual time reversal mirror, J. Appl. Phys., 084702, 109, 2011.

[99] M. Xu and L. Wang, Universal back-projection algorithm for photoacoustic computed tomography, Phys. Rev. E, 016706, 71, 2005.

[100] M. Xu and L.V. Wang, Photoacoustic imaging in biomedicine, Rev. Sci. Instr., 041101, 77, 2006.

[101] Y. Xu, L.V. Wang, G. Ambartsoumian, and P. Kuchment, Reconstructions in limited view thermoacoustic tomography, Med. Phys., 31, 724-733, 2004.

[102] L. Yao, Y. Sun, and H. Jiang, Transport-based quantitative photoacoustic tomography: Simulations and experiments, Phys. Med. Biol., 55, 1917-1934, 2010.

[103] Z. Yuan and H. Jiang, Simultaneous recovery of tissue physiological and acoustic properties and the criteria for wavelength selection in multispectral photoacoustic tomography, Optics Lett., 34, 1714-1716, 2009.

[104] Z. Yuan, Q. Wang, and H. Jiang, Reconstruction of optical absorption coefficient maps of heterogeneous media by photoacoustic tomography coupled with diffusion equation based regularized Newton method, Optics Express, 15, 18076-18081, 2007.

[105] A.D. Zacharopoulos, P. Svenmarker, J. Axelsson, M. Schweiger, S.R. Arridge, and S. Andersson-Engels, A matrix-free algorithm for multiple wavelength fluorescence tomography, Optics Express, 17, 3025-3035, 2009.

[106] R.J. Zemp, Quantitative photoacoustic tomography with multiple optical sources, Applied Optics, 49, 3566-3572, 2010.

[107] B. Zhu and E.M. Sevick-Muraca, Reconstruction of sectional images in frequency-domain based photoacoustic imaging, Optics Express, 19, 23286-23297, 2011. 